\title{
Digital rock physics in four dimensions: Simulating cementation and its effect on seismic velocity
}

\author{
J. Singh ${ }^{1,2, *}$, P. A. Cilli ${ }^{1,2}$, A. Hosa ${ }^{1,2}$, I. G. Main ${ }^{1,2}$ \\ ${ }^{1}$ University of Edinburgh, School of Geosciences, Edinburgh, UK \\ ${ }^{2}$ International Centre for Carbonate Reservoirs, Edinburgh, UK \\ * Now at: University of Strathclyde, Department of Mathematics and Statistics
}

\section{SUMMARY}

Porosity exerts a strong control on the mechanical and hydraulic properties of rocks, but can often only be imaged indirectly from the surface using geophysical measurements, such as seismic velocity. Understanding and quantifying the relationship between seismic velocity and porosity is therefore a fundamental goal of many rock physics models. Simulating the geological processes that control porosity to generate digital rocks, and numerically modelling wave propagation to estimate their elastic properties, allows for flexible and rapid calibration of velocity-porosity trends. Here, the initial deposition of two digital carbonate sediments are simulated: grainstone (near spherical grains) and coquina (anisotropic shell fragments). The gradual precipitation of cement is then simulated, resulting in a suite of 3D volumes of varying porosity with otherwise constant and known mineral and grain phases. These models are then used as input to a 3D acoustic staggeredgrid finite difference simulation of wavefield propagation, from which we estimate bulk seismic velocity and calculate the estimated bulk modulus. The resulting bulk modulus varies systematically with respect to porosity within the physical limits imposed by the Hashin-Shtrikman bounds. The samples exhibit anisotropy in the measured velocity con- 
sistent with structural anisotropy due to the settling of elongate grains under gravity. We use the resulting bulk velocity-porosity trends to test competing rock physics models, including one that accounts for varying effective pore-aspect ratio with porosity. The results validate the hypothesis that there is a power-law relationship between effective pore aspect ratio and porosity. This relationship is consistent with similar results obtained from a suite of natural carbonate grainstones examined in the laboratory. The results show the optimal rock physics model to be relatively insensitive to the degree of anisotropy in the fabric of the starting material, and may now be used with more confidence to link observed changes in effective pore aspect ratio to changes in porosity due to a range of geological processes, for example fracturing, dissolution and compaction, where other process-based models are available.

Key words: Microstructure, Numerical modelling, Computational seismology

\section{INTRODUCTION}

Geophysical properties such as seismic velocity, density and electrical resistivity depend strongly on porosity, and the form of this relationship is fundamental to applications of imaging and modelling natural processes in the solid Earth. Specific examples include the characterization and monitoring of geomechanical processes such as compaction (Zimmer 2004) or elucidating the process of rock fracturing (Pyrak-Nolte et al. 1990). Seismic velocity is often used to monitor hydraulic processes where spatio-temporal changes in the seismic velocity may be caused by fluid flow and/or changes in effective stress during subsurface injection or production (Arts et al. 2004; Brown 2002; Guilbot $\&$ Smith 2002; Stork et al. 2018). Seismic velocity is also sensitive to diagentic processes, such as cementation or dissolution (Dvorkin \& Nur 1996; Fabricius 2003; Weil et al. 2011).

Diagenesis is any physical, chemical, or biological alteration of sediments to form a sedimentary rock (Bathurst 1972; Tucker \& Wright 2009) or to alter it thereafter (sometimes called secondary diagenesis). It strongly influences reservoir quality, with strong controls on porosity (Bjørlykke et al. 1989; Moore 1989), permeability (Bloch et al. 2002; Nadeau 1998), and wettability (Barclay \& Worden 2000). Understanding diagenetic processes, and how geophysical properties relate to them, is therefore important for hydrocarbon production, $\mathrm{CO}_{2}$ injection, and groundwater resource management. However, measuring geophysical properties associated with diagenetic processes can be very challenging, even in a controlled laboratory environment. Diagenesis often occurs over very long timescales and in extreme conditions making it impractical to reproduce in the laboratory. In some 
experiments, microbial-induced calcite precipitation has been shown to accelerate cementation (Karol \& Berardinelli 2003; Saneiyan et al. 2018), but these methods require elaborate and expensive experimental apparatus. This practical problem is a prime motivation for using digital rocks, where geological or diagenetic processes such as cementation can be simulated in principle over any time scale. A digital rock is a three-dimensional representation of a rock fabric, where individual phases within a rock (e.g., each mineral component and pore space/fluid) is known for each voxel in three dimensions (see Andrä et al. (2013a,b) for an extensive review of digital rock physics). Digital rock models are far more flexible and far less expensive to run than laboratory experiments, and can be used to test hypotheses and establish trends of evolving geophysical properties including specific diagentic processes. Digital rock physics begins with a starting model from a three-dimensional image, most commonly obtained from high resolution x-ray micro-tomography, where data is segmented into individual phases (e.g., grain, pore, cement). Bulk rock properties can then be estimated, such as permeability (Martys et al. 1999; Keehm 2003), seismic velocity (Saenger et al. 2000; Saenger 2008; Arns et al. 2002), thermal conductivity (Wiegmann \& Zemitis 2006) and electrical resistivity (Liu et al. 2009; Zhan et al. 2010). Rather than the x-ray imaging of real rocks, here we simulate rock deposition and cementation to form digital rocks.

There are several examples of process-based simulation of cementation in digital rocks, including isopachous and syntaxial cementation in both sandstones (Mousavi \& Bryant 2012; Latief et al. 2010) and carbonates (Biswal et al. 2007; Mousavi et al. 2012). However, the physical properties investigated during cementation are most commonly transport properties such as permeability and electrical conductivity (e.g., Keehm et al. (2001)); to date there have been comparatively limited applications of process-based cementation modelling for the estimation of elastic properties using digital rocks (Sain 2011; Sain et al. 2014).

In order to interpret a change in the measured seismic velocity, a suitable rock physics model is required. There is an abundance of methods used in rock physics for the modelling of elastic moduliporosity or velocity-porosity data. Empirical methods, such as Eberhart-Phillips et al. (1989); Gardner et al. (1974); Han et al. (1986), are entirely based on empirical matches to trends in observed data. Thus, any inference of physical significance from using such models can be unreliable. Bounding models (Voigt 1889; Reuss 1929; Hashin \& Shtrikman 1963) and bounding average models (Hill 1952) recognize the uncertainty of elastic moduli for a given porosity and therefore give a range of moduli, where the exact value depends on geometric factors of the medium. However, these bounds can be far too broad for many practical applications. Inclusion-based models such as Eshelby (1957); Mori \& Tanaka (1973); Kuster \& Toksöz (1974); Berryman (1992) assume an often unrealistic, ellipsoidal inclusion shape embedded into a background material, but having this physics-based approach gen- 
erally leads to more intuitive interpretation and model results that more closely match real-rock scenarios. Conventionally, inclusion models assume a constant inclusion shape to characterize an elastic modulus-porosity trend; recently Cilli \& Chapman (2020) explored the potential for a variable inclusion aspect ratio related to porosity by a power law, which proved a better fit to existing elastic moduli-porosity data than models with a constant inclusion aspect ratio.

Here, we develop a process-based model for deposition and cementation in digital rocks, combined with the finite-difference simulation of wave propagation, and show that it is an effective method of developing understanding of changes in elastic properties. In addition we compare the Cilli-Chapman analytical rock physics model for a material with ellipsoidal inclusions with a commonly-used competing model. We compare these models using a statistical model selection method known as the corrected Akaike information criteria $\mathrm{AIC}_{C}$ (Akaike 1973; Hurvich \& Tsai 1989), for their ability to capture the effects of varying cementation, cement type, and initial rock fabric without over-fitting. We also investigate the effect of underlying anisotropy on the relationship between porosity and elastic properties.

In this study, we describe the method used for generating digital rocks, including the simulated deposition of the primary grains under gravity, followed by cementation. Following this we demonstrate the method of estimating the bulk seismic velocity and elastic modulus using a rotated-staggered-grid finite-difference numerical simulation (Saenger \& Bohlen 2004). We introduce the two existing inclusion models used for elastic moduli-porosity trends, and describe the method for selecting the most appropriate model $\left(\mathrm{AIC}_{C}\right)$, which is particularly important for comparing models with different numbers of model parameters. We compare results for grainstone (ellipsoidal grains) and coquina (shelly fragments) digital rocks, with two different types of cementation, and also investigate the effect of the initial structural anisotropy on the evolution of the elastic properties with cementation. Finally, we validate the model by comparing results from synthetic digital grainstone rocks with those of laboratory measured elastic data from Fournier et al. (2011).

\section{METHOD}

The methods used in this paper consist of a three part modelling approach. First is the processbased modelling of initial deposition and subsequent cementation to generate a suite of digital rocks. Anisotropy in the fabric is introduced due to the ellipticity of the clasts, and horizontal settling due to gravity. In the horizontal direction clasts are randomly oriented resulting in bulk transverse isotropy. Secondly we model wavefield propagation through each digital rock to estimate bulk elastic properties. Finally we statistically compare the modelling performance of the Cilli-Chapman model (porosity dependent inclusion aspect ratio) and the commonly used differential effective medium theory model 
(constant inclusion aspect ratio) to the measured elastic data using the corrected Akaike information criterion.

\subsection{Deposition and Cementation of Digital Rocks}

To generate digital rock models with different cement types, we follow the methodology described in detail by Hosa \& Wood (2017). The process first involves the analysis of thin-section images, so that a realistic distribution of grain shapes and sizes are used for the initial deposition. The deposition and initial packing of grains is generated in a process-based simulation of grains falling and settling on one another in a 3D space under gravity. The model allows for heterogenous shapes and sizes in the grains, modelled with normal distributions. In this study we investigate two grain shapes: ellipsoidal grains and shells, representing those of end-member carbonate facies such as carbonate grainstones and coquinas respectively. The initial rock fabrics from the resulting deposition and settling due to gravity are shown as the high porosity slices (panels a and d) in Figures 2, 3 and 4 . The initial porosity for coquina $(\phi=90 \%)$ is much higher than that of the grainstone $(\phi=38 \%$ ), which is a result of the difference in the geometries of the grains. Wadell (1932) defines the sphericity of a grain $\psi$ as: $\psi=S_{\text {sphere }} / S_{\text {grain }}$, where $S_{\text {sphere }}$ is the surface area of a sphere with the same volume of the grain, and $S_{\text {grain }}$ is the actual surface area of the grain. Deviations from $\psi=1$ (decreasing sphericity) results in higher porosity. The average sphericities for coquina and grainstone are $\psi_{C}=0.04$ and $\psi_{G}=0.14$, respectively.

After the simulation of the process of deposition, the resulting volume containing the grains is cropped and voxelized into a $300^{3}$ voxel domain. The voxel sizes are $10 \mu \mathrm{m}^{3}$ for the grainstones and $0.1 \mathrm{~mm}^{3}$ for the coquina, so the digital rock samples have the dimensions $3 \times 3 \times 3 \mathrm{~mm}$ and $30 \times 30 \times 30 \mathrm{~mm}$, respectively. We then model calcite cement growth. The model considers two different grain types: polycrystalline and monocrystalline, which develop different types of early marine calcite cement - isopachous and syntaxial, respectively (Figure 1). The syntaxial cement, which is associated with monocrystalline grains, is modelled in the shape of a parallelepiped and approximates the rhombohedral crystal form of calcite, which is a common calcite crystal form. In nature, syntaxial cement grows rapidly until euhedral crystal faces are reached (epitaxial growth) and approximate crystal form of calcite is achieved. The growth on the euhedral surfaces proceeds at a slower pace (mantle growth). Our method models the first, rapid stage of syntaxial growth. Isopachous cement, which develops on polycrystalline grains (consisting of many small crystals), is modeled as a layer of cement precipitated evenly around the grains. For both cement types, cementation progressed iteratively by adding a 1-voxel thick layer of cement in each iteration.

The modelled process of deposition and cementation for generation of digital rocks is greatly sim- 
a)

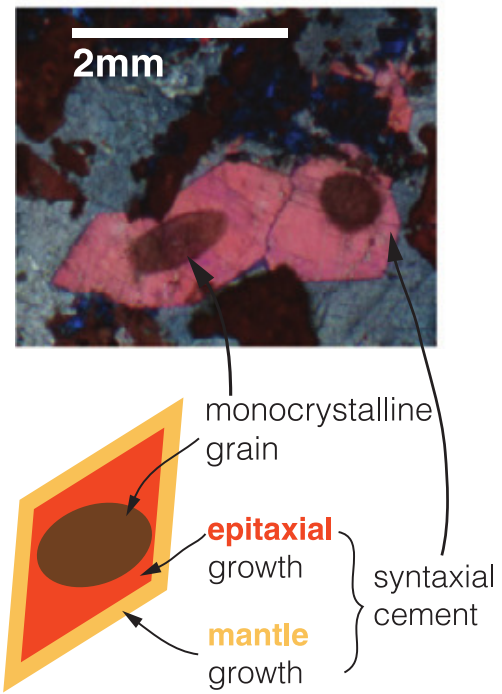

b)

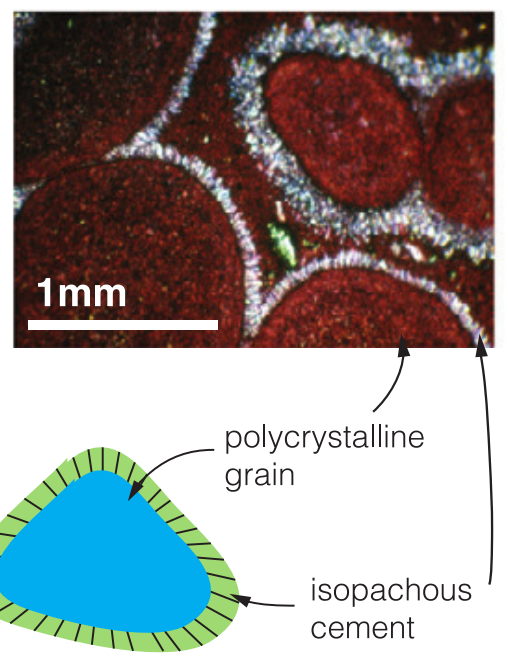

Figure 1. a) Thin-section image and schematic illustrations of syntaxial cement growth, where cement grows in the rhomobohedral crystal form of calcite, associated with monocrystalline (single crystal) grains b) Thinsection image and schematic illustrations of isopachous cement growth, where cement is precipitated evenly around polycrystalline grains. Figure adapted from Hosa \& Wood (2017).
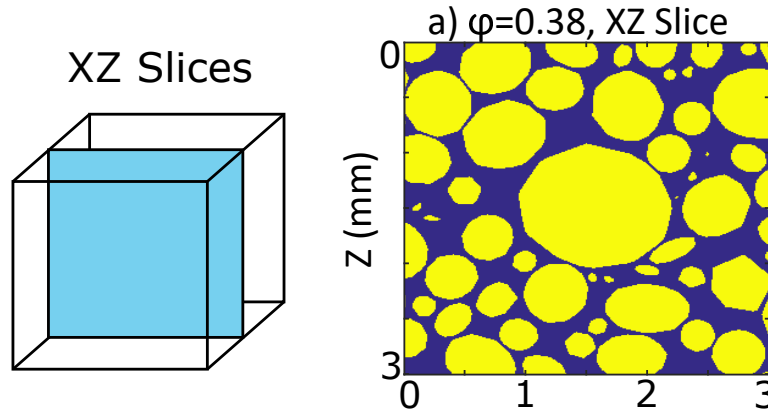

b) $\varphi=0.22, X Z$ Slice

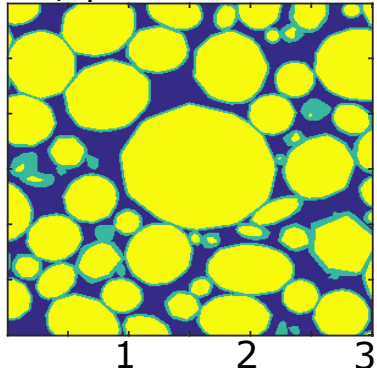

e) $\varphi=0.22, \mathrm{XY}$ Slice

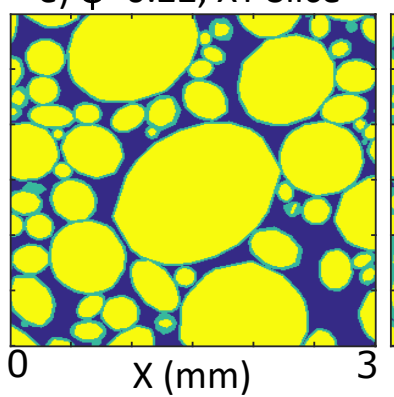

c) $\varphi=0.07$, XZ Slice

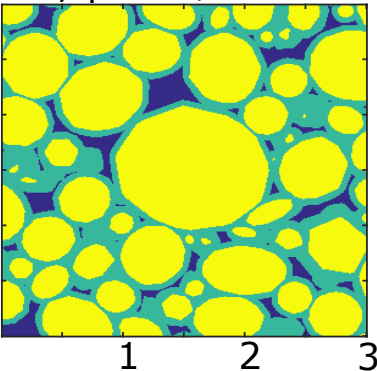

f) $\varphi=0.07, \mathrm{XY}$ Slice

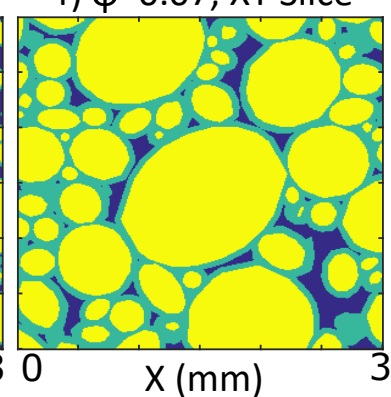

Figure 2. Example vertical (a-c) and horizontal (d-f) slices through digital rock models at varying degrees of cementation. The model uses a grainstone morphology prior to cementation, which does not change with time (yellow clasts), and a isopachous cement type that grows with time (green) into the pore space (blue). 

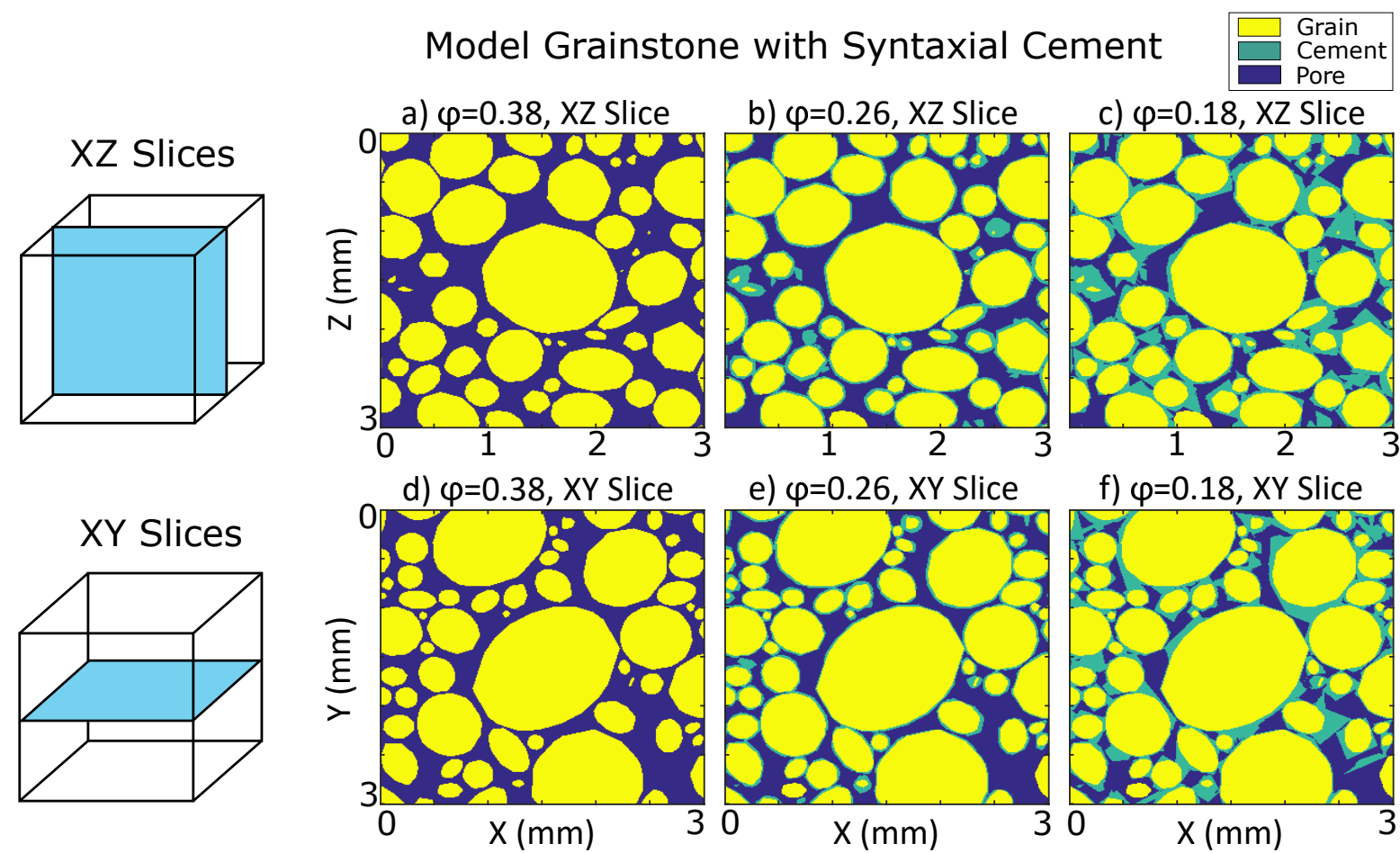

Figure 3. Example vertical (a-c) and horizontal (d-f) slices through digital rock models at varying degrees of cementation, using a grainstone morphology prior to cementation and a isopachous cement type, which does not change with time (yellow clasts), and a syntaxial cement type that grows with time (green) into the pore space (blue).

plified compared to real-world scenarios, and has several underlying assumptions: several processes are ignored such as the agitation of deposited material and re-arrangement of deposited grains due to compaction, therefore initial porosities prior to cementation are unrealistically high. This is not considered to be a problem as the focus of this study is on the change in porosity and how seismic velocity responds. The cementation method used here assumes fully saturated media, that cementation is uniform throughout the rock, and cement grows on all grains. In reality, not all pore space is accessible by percolating fluids, and cementation is linked to the fluid dynamics within the pore space. The current implementation of our method includes the continuation of precipitation in pores even after they are cut off from the percolating pore space. In reality, some sub-resolution porosity would likely allow the percolation of pore fluids but not to the extent we allow in our models.

To examine the effect of cement type, we generate a range of digital rock models using the same starting grainstone fabric assuming either polycrystalline or monocrystalline grains, therefore modeling isopachous and syntaxial cementation respectively. Representative slices through the grainstone models are shown in Figures 2 and 3. We also examine the effect of varying the initial rock deposition, where we use shell fragments representing a coquina and model isopachous cementation. 


\section{Model Coquina with Isopachous Cement}
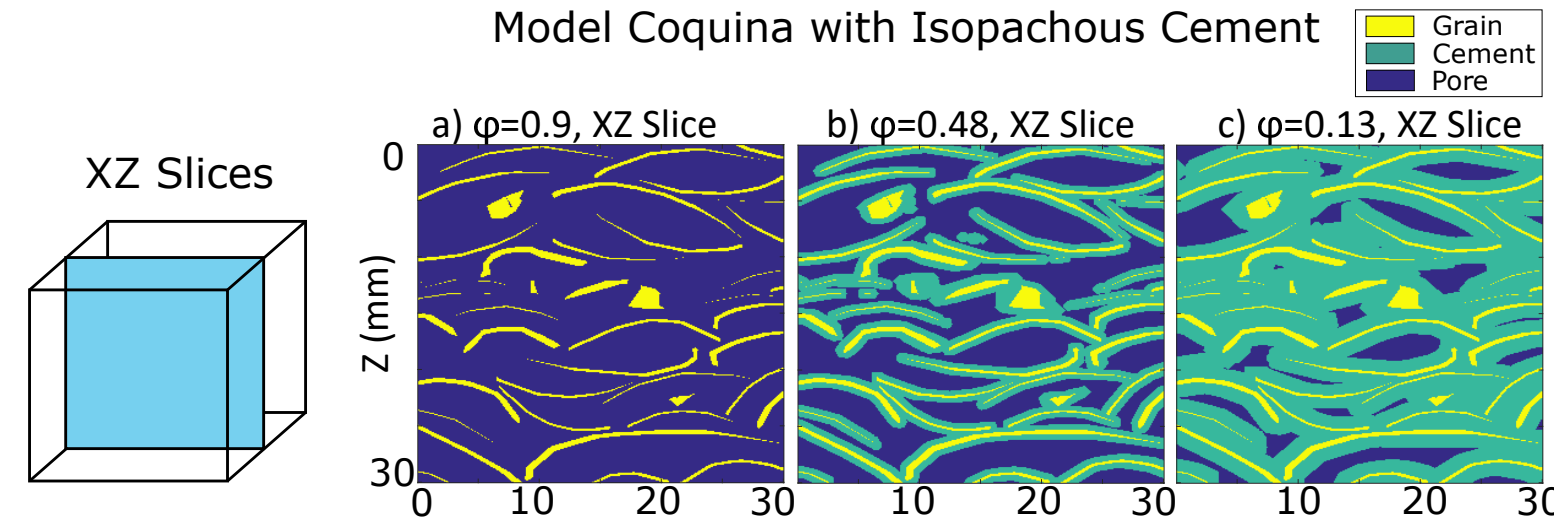

b) $\varphi=0.48, X Z$ Slice

c) $\varphi=0.13, X Z$ Slice

d) $\varphi=0.9$, XY Slice
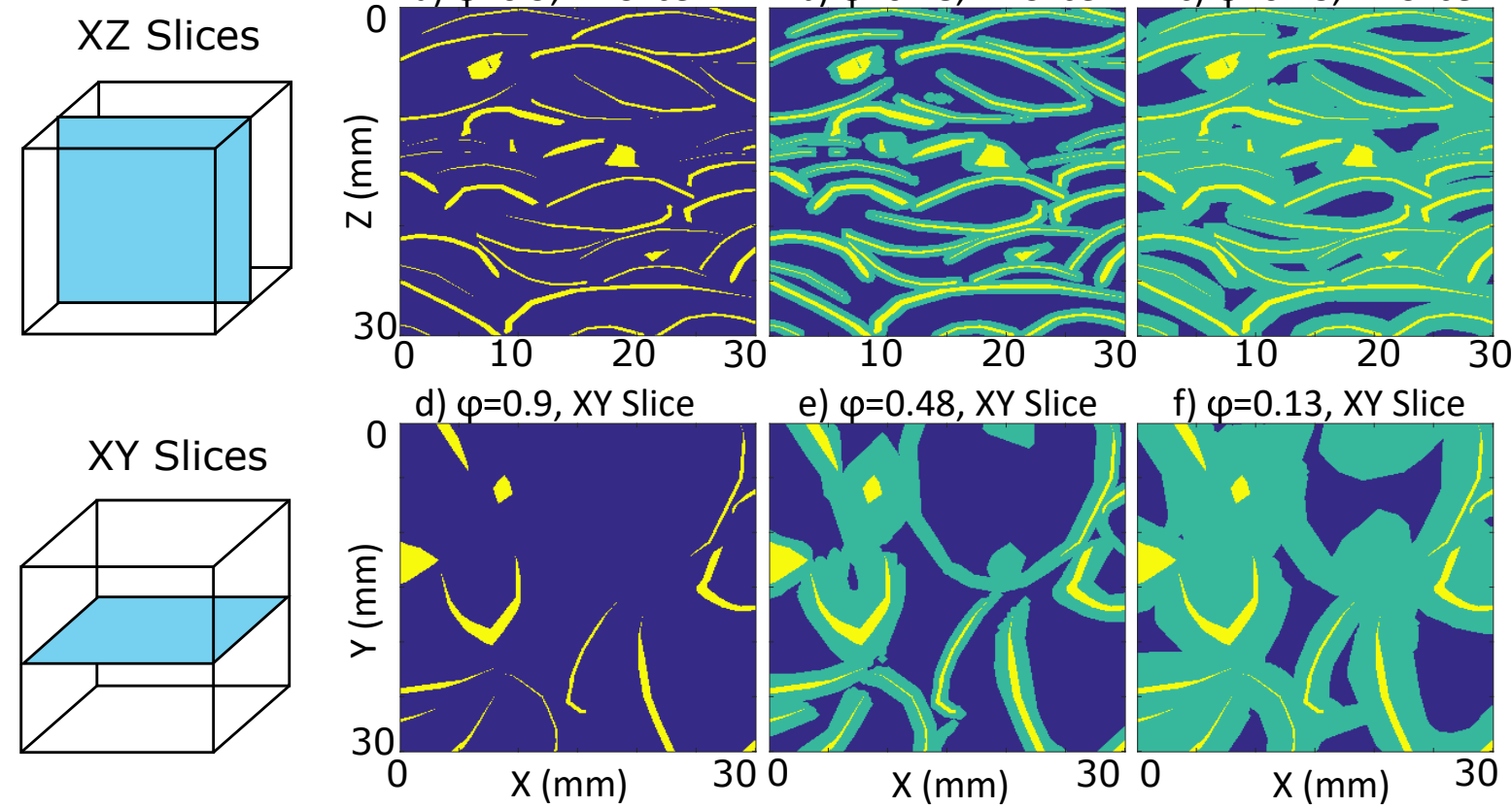

e) $\varphi=0.48$, XY Slice

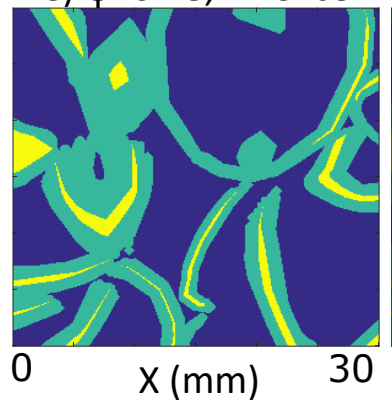

f) $\varphi=0.13, X Y$ Slice

Figure 4. Example vertical (a-c) and horizontal (d-f) slices through digital rock models at varying degrees of cementation, using a coquina morphology prior to cementation and a isopachous cement type, which does not change with time (yellow clasts), and a syntaxial cement type that grows with time (green) into the pore space (blue).

Two-dimensional slices through these models are shown in Figure 4. The sections show horizontal alignment of elongate grains, and thus some emergent anisotropy. This may be due to finite size effects here, but for larger samples we might expect transverse isotropy in the fabric due to sedimentation under gravity. We quantify the degree of anisotropy DOA (Harrigan \& Mann 1984) of the initial fabrics prior to cementation as:

$$
D O A=1-\frac{\min (\lambda)}{\max (\lambda)},
$$

where $\min (\lambda)$ and $\max (\lambda)$ are the minimum and maximum eigenvalues of the data cloud obtained by mean intercept length analysis. Mean intercept length analysis determines the number of matrix voxels that intersect with a set of oriented rays sent through the volume at different angles. The mean number of intersecting voxels as a function of angle forms the data cloud then used in eigenvalue analysis. When $D O A=0$, the fabric is perfectly isotropic, and the increases to $D O A=1$, the fabric exhibits stronger anisotropy. Both coquina and grainstone initial fabrics exhibit some emergent structural anisotropy. The details of the ranges of models for each rock type are documented in Table 1. 
Table 1. Range of Digital Rock Models, where $N$ is the number of models

\begin{tabular}{ccccc} 
Initial Fabric & Cement & $N$ & Porosity Range & Degree of Anisotropy \\
\hline Grainstone & Isopachous & 6 & $6.6-38 \%$ & 0.51 \\
Grainstone & Syntaxial & 20 & $18-38 \%$ & 0.51 \\
Shells & Isopachous & 21 & $0.1-90 \%$ & 0.55
\end{tabular}

\subsection{Estimating Elastic Properties}

The digital rock models described in Table 1, where every voxel has an assigned phase (either pore fluid, calcite grain or cement), are converted into corresponding 3D models of local velocity and density (assuming isotropic mineralogy for each phase). At this stage of the modelling we assume the properties of the precipitated cement to be the same as those of the calcite grains. The local velocity and density of calcite is taken from Mavko et al. (2009), to be $V_{P}=6500 \mathrm{~m} / \mathrm{s}$ and $\rho=2710 \mathrm{~kg} / \mathrm{m}^{3}$. We take the pore fluid velocity and density to be $V_{P}=1500 \mathrm{~m} / \mathrm{s}$ and $\rho=1000 \mathrm{~kg} / \mathrm{m}^{3}$. Each voxel is mapped to a regular grid of cells used as input to a three-dimensional finite-difference (FD) simulation of wavefield propagation (Moczo et al. 2007). The FD method discretizes the wave equation on a grid and replaces spatial derivatives by FD operators using neighboring points. High-contrast discontinuities such as those between pores and mineral phases may cause instability problems on a staggered grid. We avoid these difficulties by implementing a rotated staggered grid technique (Saenger $\&$ Bohlen 2004). We assume both point receivers and point sources as well as perfect transducer coupling, and use Ricker wavelets with central frequencies of $40 \mathrm{MHz}$ and $4 \mathrm{MHz}$ for the source time function in the grainstones and coquinas, respectively. The different frequencies used reflect the different sample sizes and resolutions of the grainstones and coquinas, so that the approximate ratio of wavelength to characteristic dimensions (i.e., grain size) is constant between the two rock types. The output is a set of synthetic seismograms from user-selected source and receiver locations. We treat these synthetic signals as if they were recorded in the laboratory, and estimate the bulk velocity from the origin time of the source signal, the arrival time of the first maximum (picked manually) and the known source-to-receiver distance. In order to measure any velocity anisotropy in the samples, we use three pairs of sources and receivers placed on opposite faces in orthogonal directions allowing for three measurements of velocity $\left(V_{P}^{X}, V_{P}^{Y}, V_{P}^{Z}\right)$. We use the empirical relationship of Pickett (1963), which linearly relates $\mathrm{P}$ and $\mathrm{S}$ wave velocities in carbonates, to estimate $\mathrm{S}$ wave velocity from the $\mathrm{P}$ wave velocity (i.e., $V_{S}=f\left(V_{P}\right)$ ). The bulk and shear moduli are then calculated using

$$
K=\rho\left(V_{P}^{2}-\frac{4}{3} V_{S}^{2}\right)
$$


and

$$
\mu=\rho V_{S}^{2},
$$

respectively. The density $\rho$ is known for each model by taking the volumetric average of densities for all voxel phases in each model. The bulk velocity lies between that of the pore fluid and the assumed local velocity of the grains and cement (Figure 5). For the coquina facies (Figure 5a) bulk velocity $V_{P}$ exhibits a significant drop at a critical porosity of $\phi_{C} \approx 30 \%$. The trend then flattens asymptotically to a lower bound, equivalent to the velocity of the pore fluid $(1500 \mathrm{~m} / \mathrm{s})$. This drop in velocity reflects a critical porosity effect; due to the thin and convex shape of the shells used coquina deposition (Fig. 4a) there are very few grain-to-grain contacts at high porosity, thus the rock is effectively fluid-supported (i.e., a suspension). As cementation increases and porosity decreases below a critical porosity $\left(\phi<\phi_{c}\right.$ ), the number of grain-to-grain contacts increases so that the rock becomes grain supported, and the velocity rapidly increases. The diagrams show bulk velocities measured in three directions. These synthetic data exhibit a drop in velocity at different porosities depending on orientation of the measurement. The initial fabric of grainstones use grain shapes which are much rounder than the shelly fragments of the coquina, and there are many grain-to-grain contacts. Consequently these digital rocks always have porosities below $\phi_{c}$ and their velocity-porosity relations exhibit a much smoother response to increasing cementation than the coquina samples (Figs. 5b and c). We see an exponential trend for isopachous cementation (Fig. 5b) and a more linear one for syntaxial cementation, which is measured over a narrow porosity range (Fig. 5c). Both grainstone samples exhibit anisotropy, where $V_{P}^{Z}$ (measured in the direction of the vertical axes in the upper panels of Figs. 2 and 3 ) is approx. $500 \mathrm{~m} / \mathrm{s}$ faster than $V_{P}^{X}$ and $V_{P}^{Y}$, as expected from the horizontal alignment in the fabric. The coquina facies also exhibits anisotropy, most notably in the critical porosity $\phi_{C}$.

\subsection{Rock Physics Modeling}

Modelled elastic data estimated from synthetic digital rocks also provides an opportunity to test and validate existing analytical rock physics models. Here we test two existing analytical rock physics models: conventional differential effective medium theory (DEMT) as of Berryman (1992), which assumes a constant pore aspect ratio, and the Cilli-Chapman (CC) model (Cilli \& Chapman 2020), which extends DEMT to include a power law relationship between porosity and pore aspect ratio. In conventional DEMT, a small volume of ellipsoidal inclusions with a distribution of aspect ratios and known elastic properties, are iteratively added into an initially homogeneous background medium with known elastic properties. After each iteration of added inclusions, the upscaled or aggregate properties of the composite are calculated and used as background medium properties for the next 

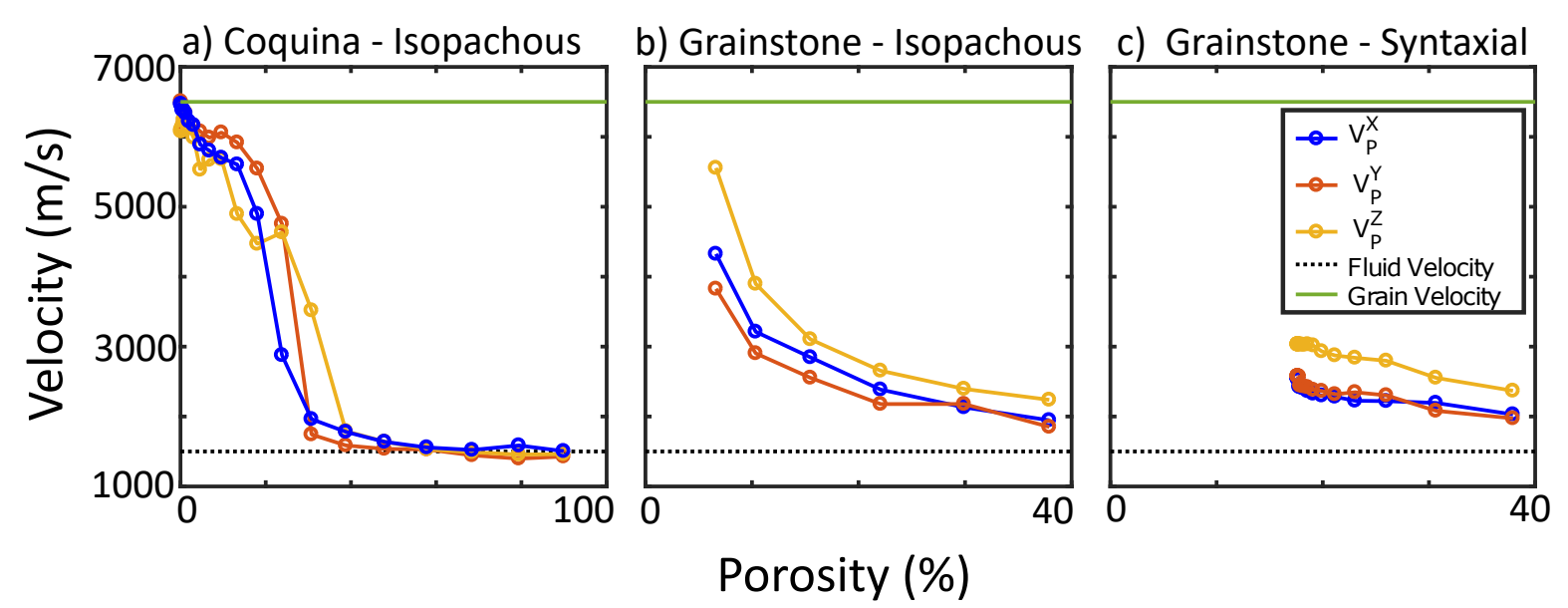

Figure 5. Bulk velocity-porosity trends measured in three orthogonal components: $V_{P}^{X}$ (blue), $V_{P}^{Y}$ (red), $V_{P}^{Z}$ (green), for a) Coquina (shelly fragments) with isopachous cementation, b) Grainstone (ellipsoids) with isopachous cementation, c) Grainstone with syntaxial cementation. The lower bound of fluid velocity (1500 m/s) and upper bound of calcite grain and cement velocity $(6500 \mathrm{~m} / \mathrm{s})$ are denoted by horizontal lines.

iteration. Letting the inclusion volume added per iteration become infinitesimally small, the inclusion volume added per iteration becomes infinitesimally small, the elastic DEMT model can be described by the following differential equations:

$$
\begin{aligned}
& (1-y) \frac{d}{d y}\left[K^{*}(y)\right]=\left(K_{2}-K^{*}(y)\right) P^{(* 2)} \\
& (1-y) \frac{d}{d y}\left[\mu^{*}(y)\right]=\left(\mu_{2}-\mu^{*}(y)\right) Q^{(* 2)}
\end{aligned}
$$

with initial conditions $K^{*}(0)=K_{1}$ and $\mu^{*}(0)=\mu_{1}$, where $K_{1}$ and $\mu_{1}$ are the inclusion bulk and shear moduli; $K^{*}$ and $\mu^{*}$ are the rock's effective bulk and shear moduli; $y$ is the inclusion volume fraction (i.e., porosity); $P$ and $Q$ are inclusion-aspect-ratio-dependent geometric factors (Berryman 1980) and their superscript denotes they are to be calculated with ellipsoids of phase 2 embedded in the effective background material. Thus, the estimated effective moduli $K_{D E M T}^{*}$ and $\mu_{D E M T}^{*}$ are functions of the elastic properties of the two phases, the porosity, and the inclusion aspect ratios. There are several assumptions and limitations to the application of the DEMT method, for example that the bulk rock is assumed to be isotropic and inclusion shapes are idealized randomly-oriented ellipsoids.

In real rocks, pores are not ideal ellipsoids, and hence the inclusion aspect ratios are simply parameters that quantify the contribution of a rock's pore space architecture to the rock's overall elastic moduli. To make this distinction between the theoretical model inclusion aspect ratios and its applied physical interpretation, Fournier et al. $(2011,2014,2018)$ and Cilli \& Chapman (2020) refer to some average of the distribution of aspect ratios $\alpha$ as the 'equivalent' pore aspect ratio (EPAR). The EPAR for a given sample can be estimated by minimising the misfit between measured bulk modulus $K_{\text {meas }}$ 
and the forward modelled moduli using Equations 4 and 5:

$$
\Psi(\alpha)=\left\|K_{\text {meas }}-K_{D E M T}^{*}\left(K_{1}, \mu_{1}, K_{2}, \mu_{2}, y, \alpha\right)\right\|_{2}
$$

The EPAR is estimated by finding the aspect ratio $\alpha$ that minimises $\Psi$, where the bulk and shear moduli of the two phases $\left(K_{1}, \mu_{1}, K_{2}, \mu_{2}\right)$ and the porosity ( $\left.y\right)$ are known.

Cilli \& Chapman (2020) investigated how a rock's effective pore and grain aspect ratios (EPAR and EGAR) changed with porosity for seven public domain datasets for elastic and electrical properties of carbonate rocks and concluded that these parameters vary with porosity as a power law, of the form:

$$
\alpha=\Gamma \phi^{\xi},
$$

where $\alpha$ represents the EPAR. In the case of elastic modelling, Equation 7 reduces to the typical DEMT model, where $\alpha=\Gamma$ and $\xi=0$.

By substituting Equation 7 into Equations 4 and 5, the resultant bulk and shear moduli, $K_{C C}^{*}$ and $\mu_{C C}^{*}$, can be calculated as functions of the bulk and shear moduli of the two phases, the porosity, and two parameters $\Gamma$ and $\xi$. The Cilli-Chapman model uses an extra model parameter than the standard, constant- $\alpha$ DEMT model, so its effectiveness cannot be compared to the standard method on the residuals alone. Cilli \& Chapman (2020) use a log-relative likelihood analysis to justify the extra parameter used in fitting the data.

Given our digitally measured velocities and porosities, as well as the known fluid and matrix elastic moduli, we inverted Equations 4 and 5 to estimate the EPAR of every rock sample. We inverted for the model parameters by minimising the misfit between each sample's measured (from Equation 2) and modelled bulk modulus. We display the inverted EPARs for each data set as a function of porosity on a log-log scale in Figure 6, and fit a line of best fit through each data set's inversion results. We calculated a close approximation $\left\{\Gamma_{0}, \xi_{0}\right\}$ of the true model parameters $\{\Gamma, \xi\}$ by the gradient and constant of these lines. As the inversion for each sample's EPAR was porosity-dependent, parameters $\left\{\Gamma_{0}, \xi_{0}\right\}$ are not necessarily equal to $\{\Gamma, \xi\}$. We thus performed a non-linear global optimisation to find the solution $\{\Gamma, \xi\}$ which minimise the $l_{2}$-norm misfit of all samples by directly substituting Equation 7 into Equations 4 and 5 with starting point $\left\{\Gamma_{0}, \xi_{0}\right\}$.

We use this inversion technique for four suites of bulk modulus-porosity data: 1) synthetically generated grainstones with syntaxial cementation, 2) synthetic grainstones with isopachous cementation, 3) synthetic coquinas with isopachous cementation, and 4) a subset of the real-rock laboratory measured grainstone data from Fournier et al. (2011). The laboratory data consists of 80 outcrop samples, all exhibiting grainstone texture and with almost pure calcitic mineralogy. There is no presence of any 
inter-granular, inter-crystalline, or moldic porosity in these samples, consistent with the definition of the grainstone end-member model examined here.

\subsection{Statistically Comparing Models}

Following from the inversions using both the constant- $\alpha$ differential effective medium theory model and the variable- $\alpha$ Cilli-Chapman model, it is then necessary to test these competing models. Here we use the corrected Akaike information criterion $\left(A I C_{C}\right)$ (Akaike 1973; Hurvich \& Tsai 1989), which is a statistical tool used for model selection based on information theory. The conventional AIC method penalises models with more modelling parameters (more complex models) and favours models with smaller misfits, thus dealing with the trade-off between data fit and model simplicity. This is necessary here because the conventional DEMT and the Cilli-Chapman models require different numbers of model parameters.

Let $k$ be the number of parameters for a model, and $\hat{L}$ be the estimated likelihood function of the model (goodness of fit). The AIC value for a particular model is:

$$
A I C=2 k-2 \ln (\hat{L}) .
$$

When the number of samples $n$ is small, AIC becomes biased to models with more model parameters (McQuarrie \& Tsai 1998). There is a risk of over-fitting as the datasets used here are relatively small. We use the corrected Akaike Information Criterion $A I C_{C}$ (Hurvich \& Tsai 1989; Cavanaugh et al. 1997) to avoid this risk, defined as:

$$
A I C_{C}=A I C+\frac{2 k^{2}+2 k}{n-k-1} .
$$

The absolute $A I C_{C}$ value generally has little indication of the validity of a model (Burnham \& Anderson 2004); rather the difference between $A I C_{C}$ values $\left(\triangle A I C_{C}\right)$ for competing models is the significant measure, where $\triangle A I C_{C}=A I C_{C}^{D E M T}-A I C_{C}^{C C}$.

Burnham \& Anderson (2003) provide a practical rule-of-thumb method for the interpretation of $\triangle A I C_{C}$ values, suggesting that: if $\triangle A I C_{C}<2$, the variable- $\alpha \mathrm{CC}$ model has "insufficient" evidence to accept as the best model; if $4<\Delta A I C_{C}<7$, the $\mathrm{CC}$ model has "good" evidence and constant- $\alpha$ DEMT has considerably less evidence as the best model; and if $\Delta A I C_{C}>10$, the variable- $\alpha \mathrm{CC}$ has "compelling" evidence to be the best model (constant- $\alpha$ DEMT has negligible evidence). Here we use an extra category for where $7<\Delta A I C_{C}<10$, the CC model has "substantial" evidence.

An additional statistical method employed here is Cook's distance, which is a measure used to identify the influence of individual data points when performing least-squares regression (Cook 1977). Cook's distance is calculated for individual data points of interest. We employ the guidelines of Burn- 
ham \& Anderson (2003) for interpreting $\triangle A I C_{C}$ values and provide all inversion results, including the output parameters $\Gamma$ and $\xi$ from the Cilli-Chapman model and $\alpha_{D E M T}$ from conventional differential effective medium theory, summarized in Table 2.

\section{RESULTS}

\subsection{Grainstone}

To test both the differential effective medium theory and Cilli-Chapman models, we first use elastic moduli estimated for grainstone samples using both syntaxial and isopachous cementation, as well as the laboratory measured grainstone data from Fournier et al. (2011). The grainstone samples with syntaxial cementation (Figures 6a and b) show a strong power-law relationship between the EPAR and porosity (plotting linearly on a log-log scale). The data points for bulk modulus clearly cross-cut the forward modeled line (dashed red curve) using DEMT, which assumes a constant aspect ratio for all porosities. The Cilli-Chapman model (variable aspect ratio) provides a significant improvement in data fit in these cases. Despite the penalty for the additional modeling parameter (therefore an additional degree of freedom), the Cilli-Chapman model outperforms DEMT in a "compelling" way $\left(\triangle A I C_{C}=51.3\right)$.

Grainstone samples using isopachous cementation shows a similar trend (Figures $6 \mathrm{c}$ and d), where there is clear variation of the EPAR with changing porosity. However, the lowest porosity sample $(\phi=6.6 \%)$ does not follow the trend exhibited by the other samples. According to $\mathrm{AIC}_{C}$, there is "good" evidence that in this case the DEMT is the best model to describe the data indicated by anegative value of $A I C_{C}\left(\triangle A I C_{C}=-3.8\right)$. The low porosity sample can be identified as 'highly influential' to the model according to Cook's distance $\left(d_{C}=2.65\right)$, well above the recommended threshold for a sample to be considered highly influential, i.e., where $d_{C}>1$ (Cook \& Weisberg 1982). The high value of $d_{C}$ does not necessarily give grounds to reject the sample from the inversion, but indicates the data point should be examined more closely, and possibly indicates the limits of using digital rock physics at such low porosities (this is further discussed in Section 4.3). For comparison, Figures $6 \mathrm{c}$ and $\mathrm{d}$ include inversion results using Cilli-Chapman model for both the full porosity range (blue) and one excluding the low porosity sample (green). Where the low porosity value is excluded $\Delta A I C_{C}=9.13$, which suggests substantial evidence for the Cilli-Chapman model as the preferred model.

Compared with the synthetic digital rock results, the EPAR-porosity relationship for the laboratory measured 'real-rock' grainstones exhibits a similar $\Gamma$ value, but a significantly lower $\xi$ value (Figs. 6e and f). When $\xi$ is small, the Cilli-Chapman model closer resembles the constant-aspect ratio DEMT 


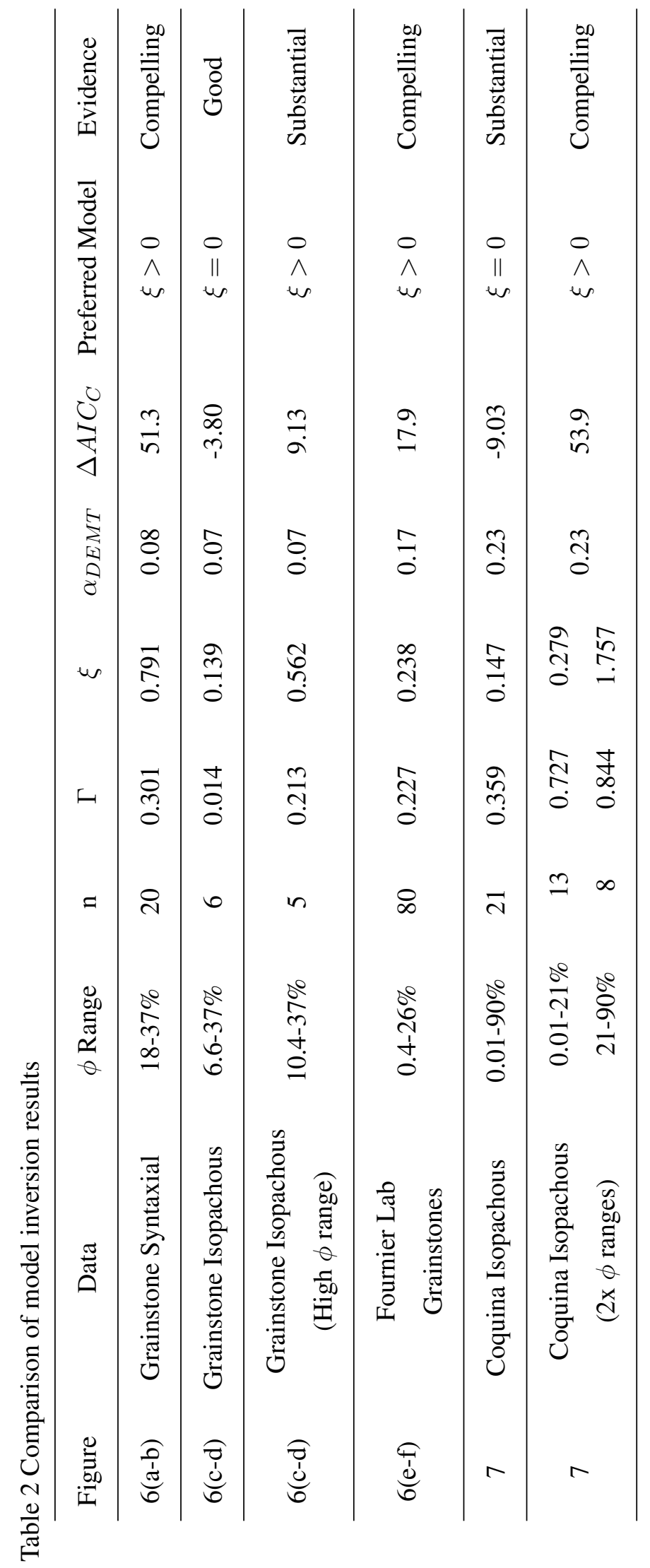


a) Grainstone Syntaxial: Bulk Modulus

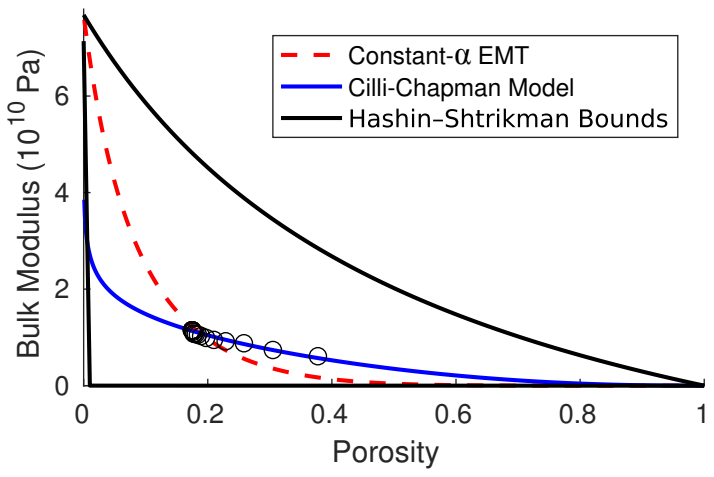

c) Grainstone Isopachous: Bulk Modulus

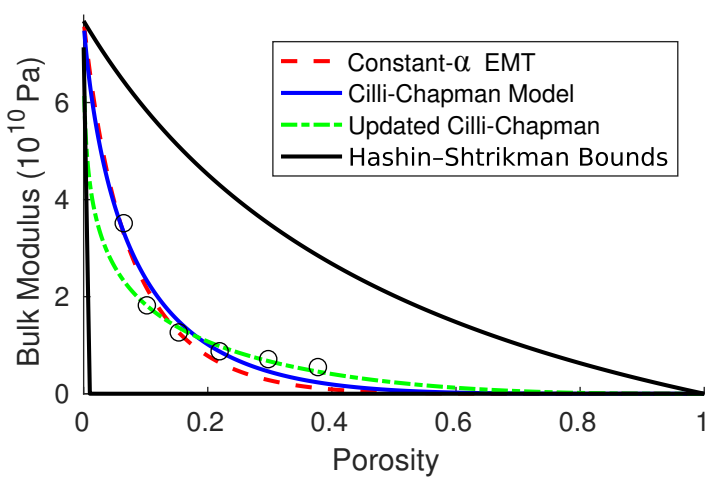

e) Fournier Lab Grainstone: Bulk Modulus

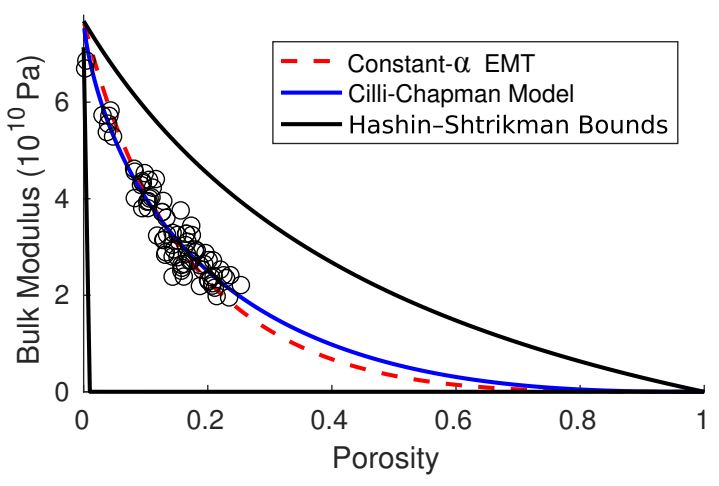

[]

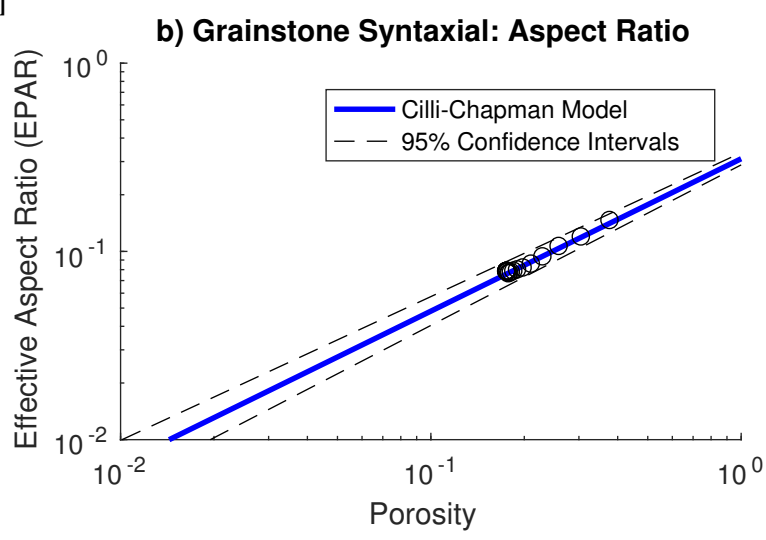

d) Grainstone Isopachous: Aspect Ratio
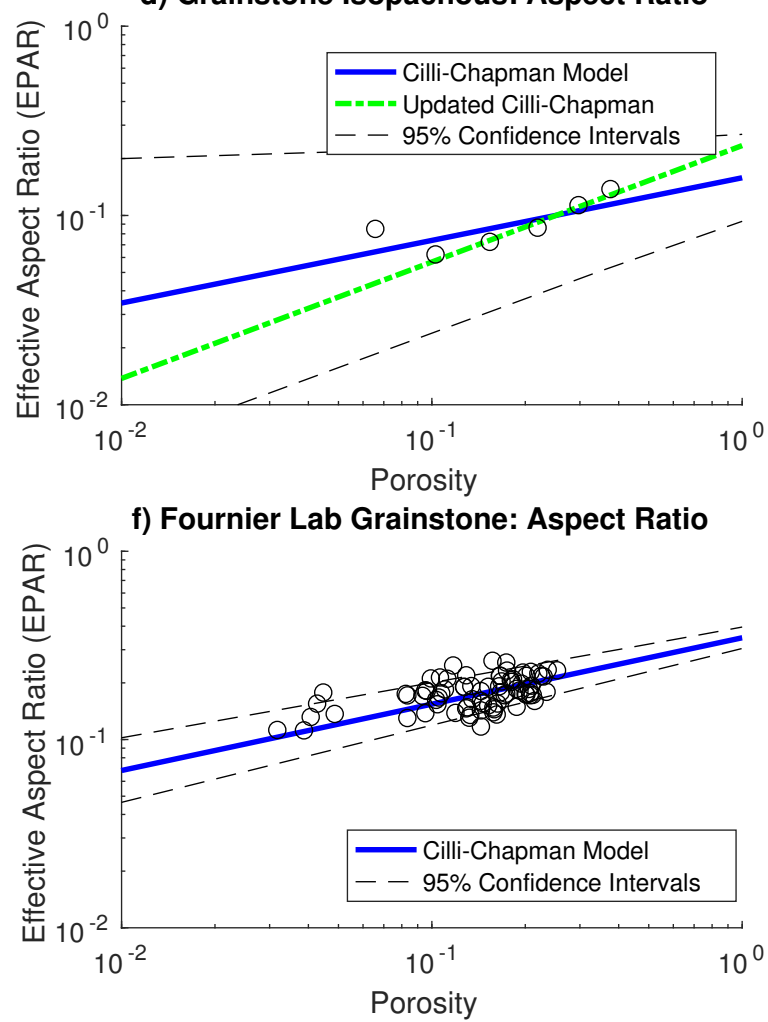

Figure 6. Rock physics modelling results for grainstone data. The left panels are the modelled bulk modulus trends using a constant- $\alpha$ DEMT model (dashed red) and the variable- $\alpha$ Cilli-Chapman model (solid blue). The right panels show inverted EPAR as a function of porosity. The figure shows results for: $a-b)$ syntaxial cementation, c-d) isopachous cementation, e-f) laboratory measured grainstone data from Fournier et al. (2011). For the isopachous cementation data (c-d), we include an updated model (green), where the low porosity is rejected in the regression as the data point has Cook's distance $d_{C}$ equal to 2.650 , well above the recommended threshold $d_{C}>1$ (Cook \& Weisberg 1982). 


\section{Single Orientation (Z)}
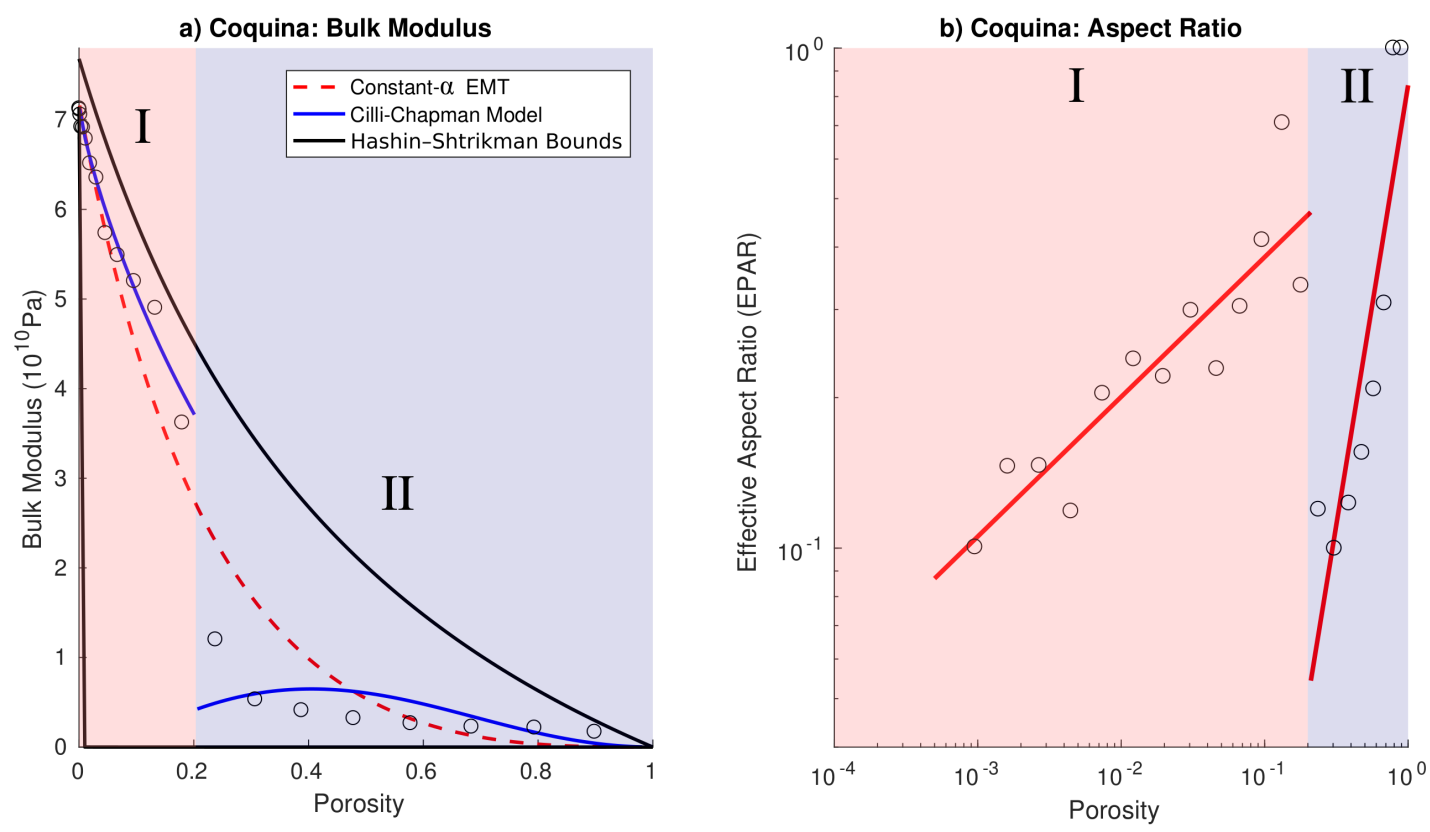

Figure 7. Rock physics model comparison for Coquina samples, using bulk modulus inferred from velocities measured perpendicular to shell orientation ( $\mathrm{Z}$ direction, vertical direction in Figures $4 \mathrm{a}-\mathrm{c}$ ). a) Bulk modulusporosity trend for a constant- $\alpha$ DEMT model (dashed red) and the variable- $\alpha$ Cilli-Chapman model (solid blue) separated into two separate porosity ranges (critical porosity $\phi_{c}=0.21$ ) with two power-law relationships. b) Inverted aspect ratio $\alpha$ (circles) and two linear trend-lines: I) $\phi<0.21$ and II) $\phi>0.21$. Porosity range I is below critical porosity $(\phi<0.21)$ and exhibits the previously observed power-law (linear on $\log$-log scale) relationship. Stage II is above critical porosity $(\phi>0.21)$, where inverted aspect ratio values drop initially, then increase to $\alpha=1$.

model (Equation 7). Despite the small variation in models, the improvement in data fit is regarded as "compelling", where $\triangle A I C_{C}=17.9$, due to the large number of data points. The difference between digital rock simulations and laboratory results is discussed in Section 4.2.

\subsection{Coquina}

The bulk modulus and the inverted Equivalent Pore Aspect Ratio (EPAR) values for the Coquina samples, estimated using the same method as in Section 3.1, are shown in Figure 7. This data is for velocity measurements taken only in the direction perpendicular to shell orientations ( $V_{P}^{Z}$ in Figure 5a, and the vertical direction in Figures $4 \mathrm{a}-\mathrm{c})$. The coquina samples contain a very large porosity range $(\phi=0.01-0.90)$, and exhibit much more complex pore shapes in comparison with those in the grainstone samples. There appears to be two distinct EPAR-porosity trend-lines, separated into stages: I) $\phi<0.21$ and II) $\phi>0.21$ in Figure 7b. This is interpreted as a critical porosity effect, where above 
a given porosity $\phi_{C}$ the rock can be treated as fluid supported, which also explains the abrupt drop in bulk modulus where $\phi>0.21$ in Figure 4a. Below this threshold the rock is grain or clast-supported. When the full range of porosity samples are included in the inversion the constant- $\alpha$ DEMT model is statistically much stronger ( $\triangle A I C_{C}=-9.03$ ) than a single power law relationship. However, when two separate inversion are performed for porosities both above and below $\phi_{C}=0.21$, where two power law relationships doubles the number of model parameters, $\Delta A I C_{C}=53.9$, which suggests compelling evidence for the two stage Cilli-Chapman model as the most appropriate.

The EPAR of a rock is related to its solid frame's stiffness. When $\alpha=0$, the frame is as weak as possible and the rock's moduli coincide with the lower Hashin-Shtrikman (Hashin \& Shtrikman 1963) and Reuss (Reuss 1929) bounds (Norris 1985). A fluid-filled porous rock with a measured porosity above its critical porosity behaves in a similar way to a suspension of solid material in a background of fluid and has elastic moduli coinciding with the lower Hashin-Shtrikman bound. In the case of the Coquina samples (Figure 7), we see the rock's EPAR increasing with respect to the HashinShtrikman bounds as porosity increases in stage I, implying the matrix is becoming relatively more stiff as porosity increases. At the interface between stage I and stage II, however, the inverted EPARs plummet as the rock passes its critical porosity and becomes significantly softer. In stage II the rock is acting approximately as a suspension. The Coquina's measured bulk modulus does not go exactly to the Hashin-Shtrikman lower bound, but rather seems to stay at an approximately constant value for all porosities above the critical porosity (Fig. 7). The method used for generating digital rocks involves the deposition of falling grains and settling due to gravity, where grain-to-grain contacts must be present. Therefore the media cannot be true suspensions under gravity, though a critical porosity effect is still observed during dynamic wave propagation. Even at $90 \%$ porosity, some load-bearing connected paths (force chains) exist through the matrix material from one side of the digital rock to the other under gravity (Fig. 4). If connected paths like this exist over all porosities, then the bulk modulus will be higher than the Hashin-Shtrikman lower bound (Fig. 7). The bulk modulus remains approximately constant for the high porosity samples, while the upper and lower Hashin-Shtrikman bounds converge with increasing porosity. The bulk modulus is hence becoming closer to the upper Hashin-Shtrikman bound with increasing porosity in stage II, which is the cause of the observed increasing EPAR with porosity in this stage. The observed critical porosity is discussed further in Section 4.4.

\subsection{Anisotropy}

The velocity of the intital grainstone fabric measured in the $\mathrm{Z}$ direction (vertical direction in Figures 3 and 2a-c) is approximately $500 \mathrm{~m} / \mathrm{s}$ faster than $V_{P}^{X}$ and $V_{P}^{Y}$ (Figs. $5 \mathrm{~b}$ and c). To further understand the effect of anisotropy on the rock physics model inversions, we invert for the independent EPAR pa- 


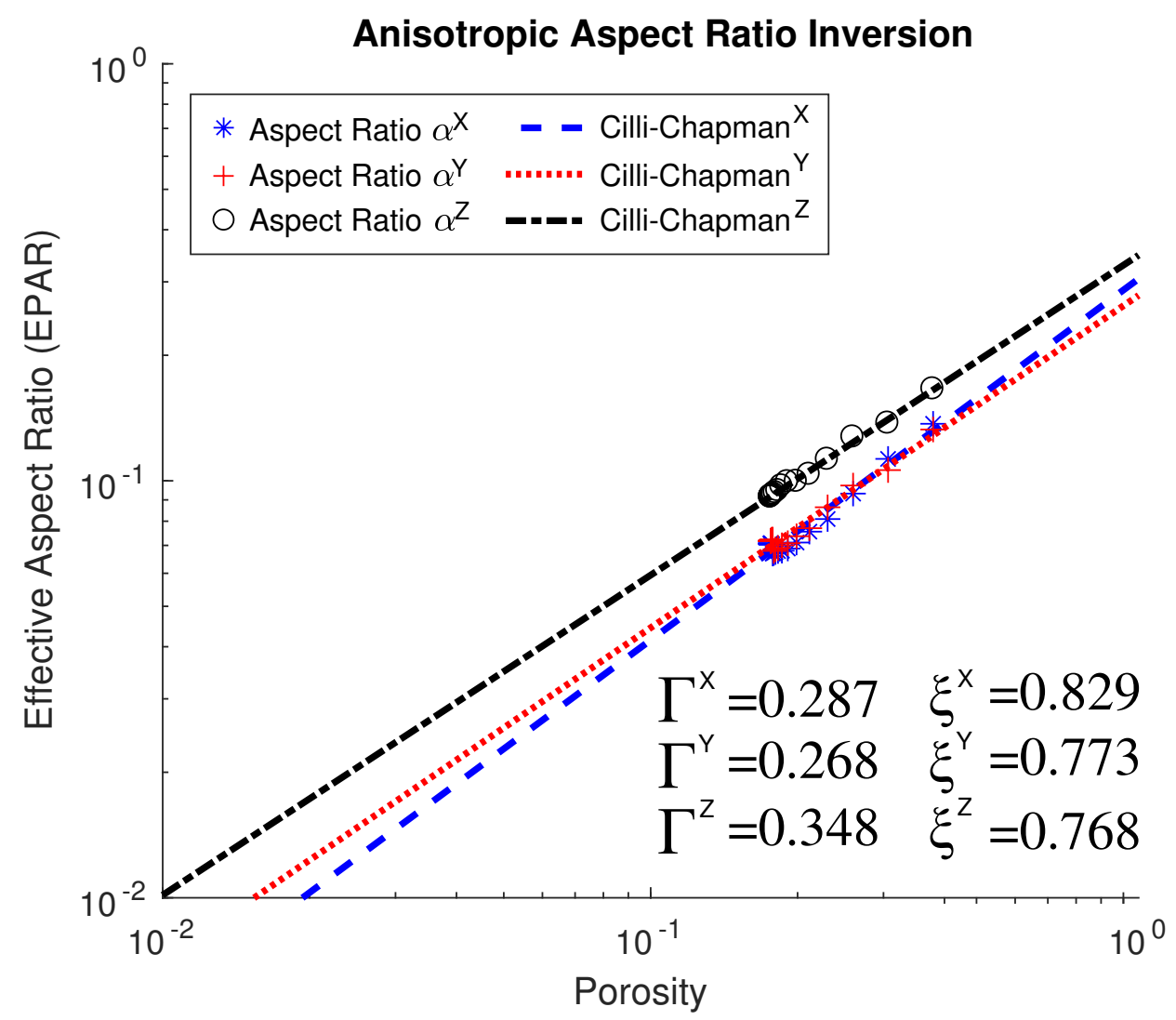

Figure 8. Inverted aspect ratio $\alpha$ as a function of porosity, for grainstones with syntaxial cementation. Measurements are made using elastic data measured in three orthogonal directions (the input velocity measurements are shown in Figure 5c). $\Gamma$ (aspect ratio $\alpha$ at the porosity $\phi=1$ intersect) and $\xi$ (gradient of the trend-line on log-log scale) for each orientation are labelled.

rameters $\left(\alpha_{X}, \alpha_{Y}, \alpha_{Z}\right)$ using bulk modulus calculated from the three separate orientations, using the grainstone samples with syntaxial cementation. The resulting inverted EPAR as a function of porosity are shown in Figure 8. The results are consistent with a bulk seismic anisotropy that is transversely isotropic, where $V_{P}^{Z}>V_{P}^{X} \approx V_{P}^{Y}$ leading to $\alpha^{Z}>\alpha^{X} \approx \alpha^{Y}$ at all porosities in Figure 8. The observed anisotropy is consistent with the input model fabric generated by sedimentation under gravity. Interestingly the effect of anisotropy is only on the $\Gamma$ parameter in the Cilli-Chapman model (the theoretical inclusion aspect ratio $\alpha$ where porosity $\phi=1$ ), whereas $\xi$ (the gradient of the trend-line on a log-log scale) is approximately constant, independent of orientation and anisotropy. In this case, the estimated $\xi$ parameter can be assumed to be representative of the entire medium, so can be used if the bulk modulus is inferred from measured velocity along a single available orientation (as is often the case in laboratory experiments). 


\section{DISCUSSION}

\subsection{Validity of high-frequency numerical data}

The data simulated here uses numerical finite-difference methods that are in the high frequency (short wavelength) regime. However, effective medium theory is generally derived for the zero-Hertz limit (i.e., static loading). Therefore, high frequency ultrasonic data should be interpreted with caution when using such methods that are better suited to long wavelength field seismic data. Batzle et al. (2006) show that when fluids are present, measurement for seismic velocity can strongly vary between low and high frequencies. In these high frequency cases, such as the data we present here, various poroelastic inclusion models could be considered (e.g., Chapman (2003)). However, the synthetic data generated in this paper uses acoustic simulations, so the effects of poroelasticity and fluid mobility can be neglected and simpler models can be used. Another problem arising from performing experiments in the high frequency domain is that the recorded first arrivals are biased to the single fastest path between the source and receiver, and therefore likely to be not representative of the bulk sample. For future work, such methods could be incorporated with spatially averaging methods, such as coda wave interferometry (Singh et al. 2019), to overcome this bias. Despite the limitations mentioned here, the results we show are broadly comparable to results from low frequency real rock laboratory experiments observed in Figure 6 and overall the preference for the Cilli-Chapman model is statistically compelling (Table 2).

The Cilli-Chapman model and DEMT are constructed assuming a background medium, with spheroidal inclusions with a given aspect ratio $\alpha$ embedded within. Therefore using either DEMT or Cilli-Chapman models for suspensions, where the porosity is higher than the critical porosity $\phi_{C}$, does not follow any physical intuition and does not allow for physical interpretation. It is likely to be more appropriate to consider a separate rock physics model for suspensions (e.g., Wood (1941)) in stage II.

\subsection{Comparison of modelled and real data}

When comparing the synthetic data from modelled digital rocks and those of the 'real' laboratory measured grainstones, they appear to be similar to the first order. Both data sets exhibit a powerlaw relationship between porosity and EPAR with similar values of $\Gamma$ and both positive values of $\xi$ (see Table 2). To the second order, differences between the inverted power-law relationships are observed (Figure 6), particularly the values of $\xi$ (in Equation 7). This value denotes the gradient of the aspect ratio-porosity trend-line on a log-log graph, and reflects the sensitivity of the EPAR to porosity changes. We interpret the high $\xi$ values for digital rocks to be a product of considering 
cementation to be the only process occurring, essentially ignoring other physical effects. This results in much smoother trends with lower variance in the synthetic data compared to the data from real rocks. As porosity increases, the digital rocks show increasing EPAR behaviour, meaning the rock frame becomes relatively stiffer. However in reality, other physical effects also operate as porosity increase, such as the coupling and stiffness of grain contacts. Rocks with high porosity can be poorly consolidated, these samples would exhibit relatively low stiffness, therefore a low inverted EPAR. These different processes occurring and acting against each other essentially reduce the sensitivity of the equivalent pore EPAR to porosity (reducing $\xi$ ). A digital rock model incorporating changes in both cementation and grain contact stiffness could overcome this observed difference, but we leave this for future work.

\subsection{Digital rocks with low porosity}

In real rocks, pore structures are found to be fractals, i.e., the geometric features of the pore space are similar across all scales (Rahner et al. 2018). At the smallest scales the estimation of physical properties of porous rocks are hampered by the resolution of the 3D images, mainly due to the presence of unresolved microporosity. In digital rocks that attempt to emulate real ones, the smallest possible pore is equivalent to a single voxel with a cubic shape. Therefore digital rocks cannot replicate the fractal dimensions of a real rock, and particularly at low porosities, the pore shapes become unrealistic. This could be an explanation for the outlier result for the low porosity sample $(\phi=6.6 \%)$ of the grainstones using isopachous cementation seen in Figures 6e and d, which exhibits an EPAR higher than expected assuming a power-law relationship. As porosity decreases due to cementation, individual pore shapes become more cubic (closer to a sphere where $\alpha=1$ ) and therefore relatively stiff. The inversion results from the laboratory measured grainstones (Figures 6e and f) do not show this increase of EPAR at lower porosities indicating the phenomenon is an artifact of the finite bandwidth scaling of the digital rock. This can somewhat be overcome by increasing the resolution of the digital rocks, therefore increasing the porosity range where digital rock microstructure remains valid.

\subsection{Critical porosity}

Digital rock physics provides an insight into the elastic properties of media that either do not often occur in nature, or would be challenging to produce in laboratory experiments, such as the very high porosity ( $\phi=0.9$ ) of the initial coquina model prior to cementation. The ability to generate such high porosity models allows the observation of critical porosity effects associated with a transition from grain-supported to fluid-supported media during dynamic wave propagation. However, grain-to-grain contacts exist across all porosities, though a critical porosity effect is still observed. To explore this 


\section{J. Singh et al}

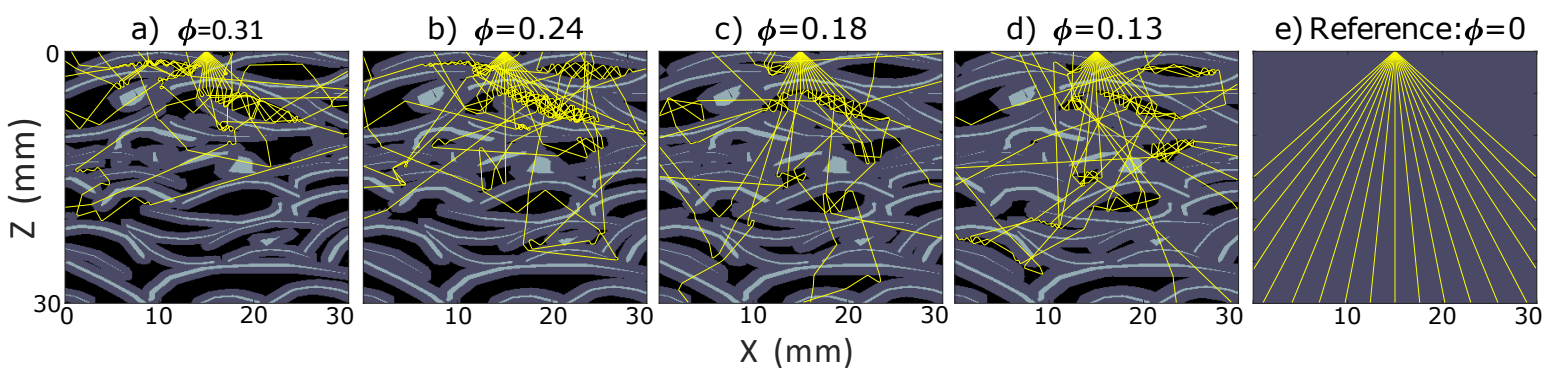

Figure 9. 2D ray tracing (Margrave 2000) results using the vertical slices through the center of coquina models with increasing cementation, over the porosity range that exhibits an abrupt increase in seismic velocity. Rays originate from the center-top of the models, and are shown for a $90^{\circ}$ aperture with $5^{\circ}$ intervals. Rays terminate when they leave the bounds of the velocity model.

further we take vertical slices through the center of four volumes, over the porosity range that exhibits the abrupt change in bulk modulus. We use the slices, converted into velocity models (following the same method described in Section 2.2), as input into the ray-tracing software of Margrave (2000). This method takes a fan of rays from a user-selected source location, and calculates the ray path, which terminates when it leaves the bounds of the model. This process allows the illustration of the difference in ray path complexity, rather than calculate absolute ray paths, as our finite-difference simulations are not limited to a 2D plane. At high porosity (Figure 9a) waves take paths with many internal reverberations. For a ray to traverse the full length of the medium (necessary for the measurement of seismic velocity with conventional experimental geometries) would require to take a complex path through grain and cement, or travel through a large amount of pore fluid. In the low porosity model (Figure 9d) rays are transmitted through the full length of the model, and paths are considerably simpler (fewer reflections) than for the higher porosity models (Figs. 9a and b). Therefore the critical porosity reflects the transition between direct tortuous paths between the source and receiver on opposite sample boundaries. Independently, Walker et al. (2016) observe a step increase in the number of force chains at a critical stress when simulating simple shear in 3D media. In principle, this could provide another possible explanation for the critical porosity effect observed in the coquina, if it can be demonstrated to occur also under static gravitational load.

\section{CONCLUSION}

The results of estimated velocity of digital rock carbonates presented here show realistic bulk modulusporosity trends for a range of carbonate morphologies and cementation types. The samples exhibit anisotropy in the measured velocities (and elastic moduli) due to structural anisotropy caused by the settling of elongate grains under gravity. Our results also highlight a significant critical porosity effect 
for the anisotropic 'shelly' fragments between phases that provide more direct or more tortuous path between the source and receiver at different stages of cementation, and possibly a significant change in the morphology of the force chain network under gravity.

For modelling bulk modulus-porosity data, the evidence for the variable-aspect ratio model of Equation 7 is statistically compelling, when compare to the conventionally used single-aspect ratio differential effective medium (DEMT) model for both grainstone and coquina digital rocks. The variable-aspect ratio model also remains robust to anisotropy where the conventional DEMT method fails. The results from simulated data and real laboratory measured data for grainstones are in good agreement, suggesting the digital rock physics framework is a valid approach for model validation. By employing the methods presented here, and other process based models such as those for fracturing or compaction, a wide range of geological processes and their relationship with elastic properties can be investigated.

\section{ACKNOWLEDGMENTS}

The authors would like to thank Petrobras and Shell for their sponsorship of the International Centre for Carbonate Reservoirs (ICCR), and for permission to publish this work from the 4DRP, VSP, and RockType II projects. We also thank Rachel Wood, Mark Chapman and Satyan Singh for their helpful scientific input and support.

\section{APPENDIX: FINITE DIFFERENCE MODEL DETAILS}

Digital rocks in this case are monomineralic (only calcite), therefore can be computed and stored as binary matrices. The local velocity and density of calcite is taken from Mavko et al. (2009), to be $V_{P}=6500 \mathrm{~m} / \mathrm{s}$ and $\rho=2710 \mathrm{~kg} / \mathrm{m}^{3}$. We take the pore fluid velocity and density to be $V_{P}=1500$ $\mathrm{m} / \mathrm{s}$ and $\rho=1000 \mathrm{~kg} / \mathrm{m}^{3}$. For each digital rock, voxels are mapped to a regular grid of cells. Some example discretised models are shown with source locations for the grainstone exhibiting isopachous cementation in Figure 10. The voxel sizes correspond to $10 \mu \mathrm{m}^{3}$ for the grainstone samples and 0.1 $\mathrm{mm}^{3}$ for the coquina samples.

We implement a rotated staggered grid acoustic finite difference simulation with reflecting boundary conditions (Saenger \& Bohlen 2004). We assume both point receivers and point sources as well as perfect transducer coupling, and use Ricker wavelets with central frequencies of $40 \mathrm{MHz}$ and $4 \mathrm{MHz}$ for the source time function in the grainstones and coquinas, respectively (the $40 \mathrm{MHz}$ source wavelet is shown in Figure 12a). The time step in the simulation is very short $\Delta t=5 \times 10^{-11} \mathrm{~s}$. The values for grid spacing $(\Delta x, \Delta y, \Delta z)$ and and time steps $(\Delta t)$ are selected to suit spatial and temporal stability 


\section{J. Singh et al}
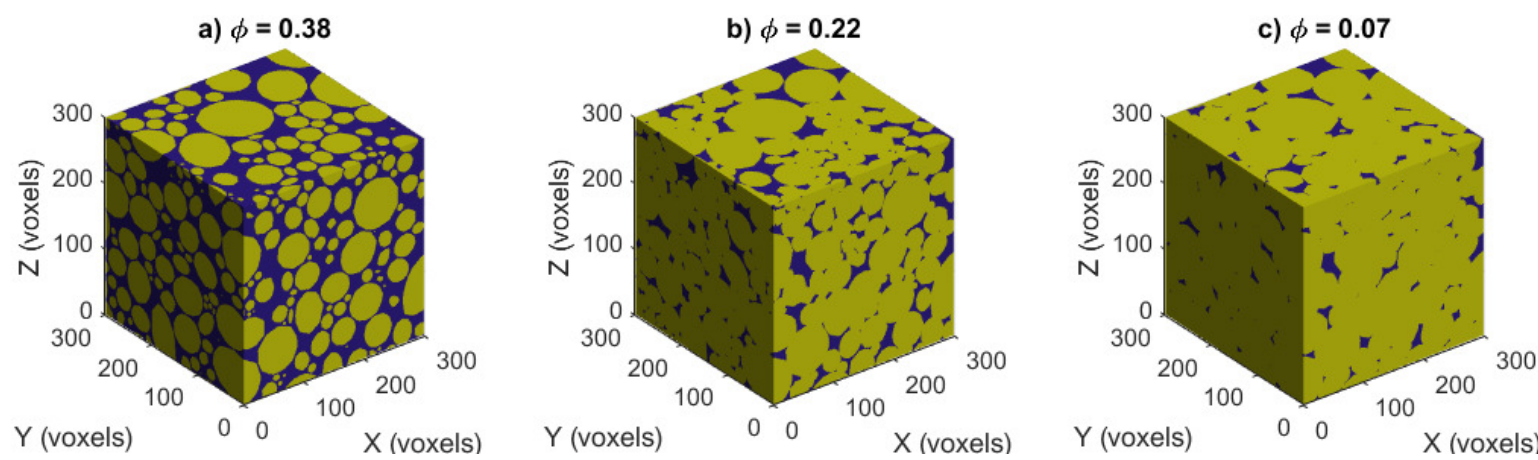

Figure 10. Example discretised models for the Grainstone digital rocks exhibiting isopachous cementation for three stages in the porosity evolution: a) $38 \%$, b) $22 \%$, c) $7 \%$. The white stars denote the three source positions used for each sample. As we are using the pulse-transmission approach, three receivers are placed on the opposite faces of the sources.

conditions:

$$
\Delta x, \Delta y, \Delta z \leq \frac{\lambda}{20}
$$

and

$$
\Delta t \leq \frac{1}{v \sqrt{\frac{1}{\Delta x^{2}}+\frac{1}{\Delta y^{2}}+\frac{1}{\Delta z^{2}}}}
$$

Example time slices of wave propagation through three grainstone models is shown in Figure 11 and the output is a set of synthetic seismograms (Figure 12).

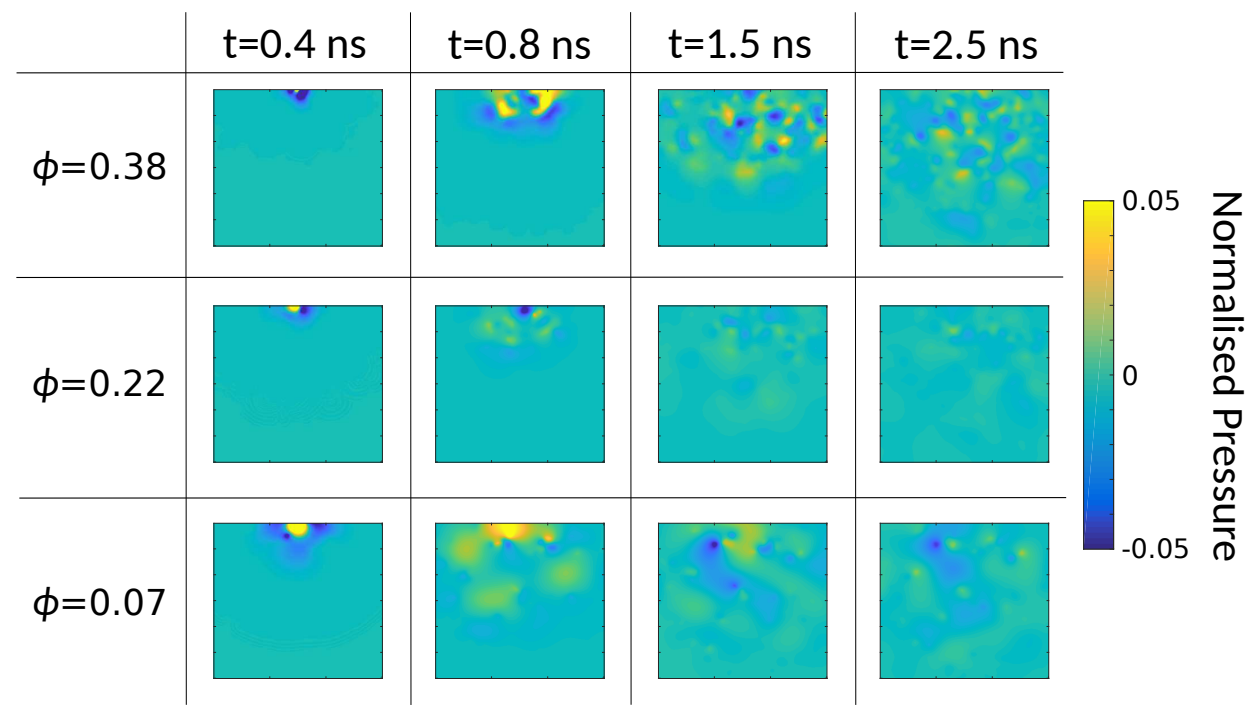

Figure 11. Example time slices of wavefield propagation through three Grainstone models (shown in Fig. 10). A slice is taken through the $\mathrm{X}-\mathrm{Z}$ plane at the same time for each sample, shown in each column. The pressure is normalised to the maximum pressure of the input wavelet. 

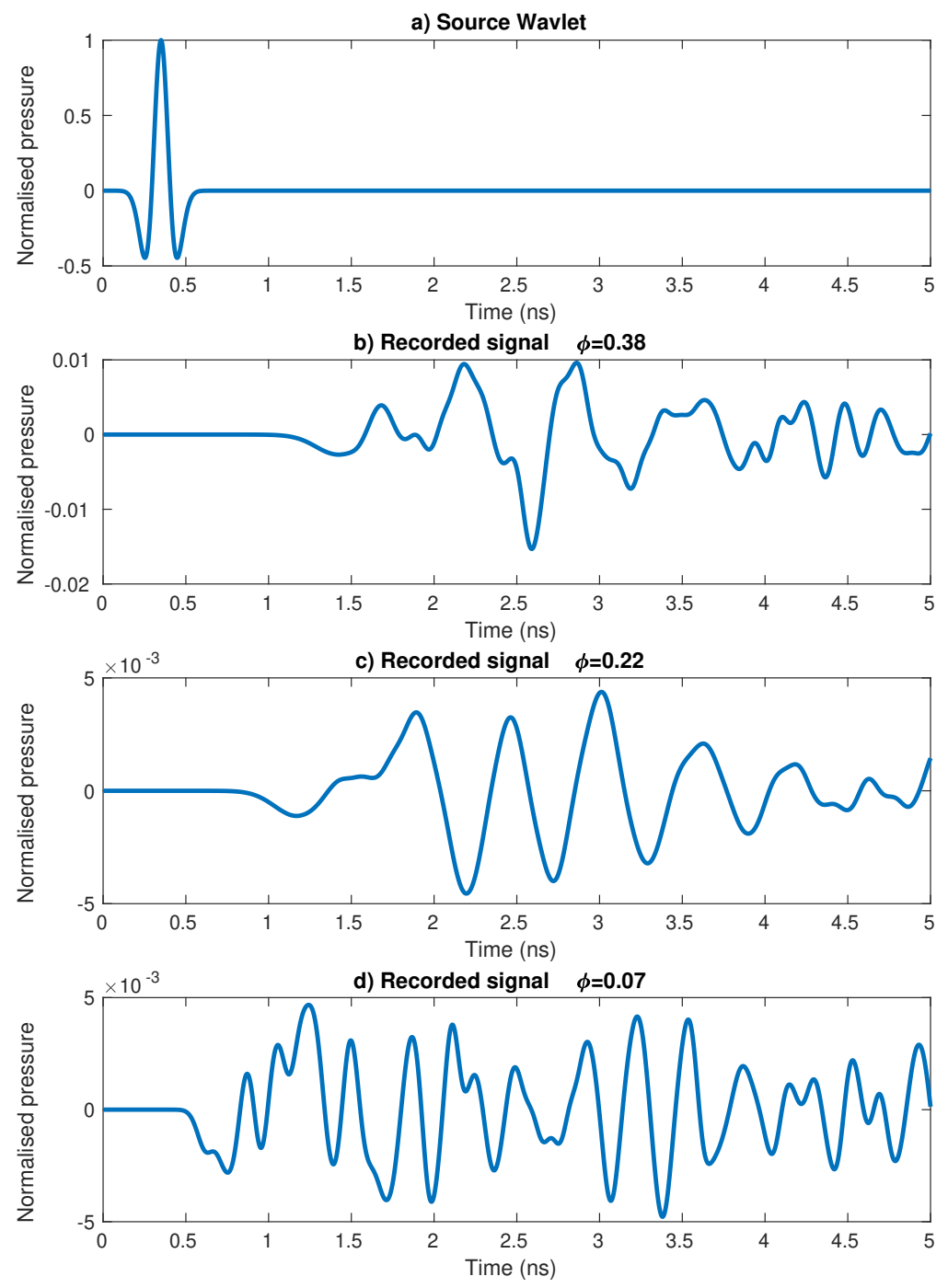

Figure 12. a) Input source wavelet used for Grainstone samples. b-d) Recorded signals along the $\mathrm{Z}$ axis for Grainstones exhibiting isopachous cementation at: a) $\phi=0.38$, b) $\phi=0.22$, c) $\phi=0.07$. In all subplots, pressure is normalised to the maximum pressure of the input wavelet. 


\section{REFERENCES}

Akaike, H., 1973. Maximum likelihood identification of gaussian autoregressive moving average models, Biometrika, 60(2), 255-265.

Andrä, H., Combaret, N., Dvorkin, J., Glatt, E., Han, J., Kabel, M., Keehm, Y., Krzikalla, F., Lee, M., Madonna, C., et al., 2013a. Digital rock physics benchmarks — part II: computing effective properties, Computers \& Geosciences, 50, 33-43.

Andrä, H., Combaret, N., Dvorkin, J., Glatt, E., Han, J., Kabel, M., Keehm, Y., Krzikalla, F., Lee, M., Madonna, C., et al., 2013b. Digital rock physics benchmarks - Part I: Imaging and segmentation, Computers \& Geosciences, 50, 25-32.

Arns, C. H., Knackstedt, M. A., Pinczewski, W. V., \& Garboczi, E. J., 2002. Computation of linear elastic properties from microtomographic images: Methodology and agreement between theory and experiment, Geophysics, 67(5), 1396-1405.

Arts, R., Eiken, O., Chadwick, A., Zweigel, P., Van der Meer, L., \& Zinszner, B., 2004. Monitoring of CO2 injected at Sleipner using time-lapse seismic data, Energy, 29(9-10), 1383-1392.

Barclay, S. \& Worden, R., 2000. Effects of reservoir wettability on quartz cementation in oil fields, Quartz Cementation in Sandstones, pp. 103-117.

Bathurst, R. G., 1972. Carbonate sediments and their diagenesis, vol. 12, Elsevier.

Batzle, M. L., Han, D.-H., \& Hofmann, R., 2006. Fluid mobility and frequency-dependent seismic velocity—direct measurements, Geophysics, 71(1), N1-N9.

Berryman, J. G., 1980. Long-wavelength propagation in composite elastic media II. ellipsoidal inclusions, The Journal of the Acoustical Society of America, 68(6), 1820-1831.

Berryman, J. G., 1992. Single-scattering approximations for coefficients in Biot's equations of poroelasticity, The Journal of the Acoustical Society of America, 91(2), 551-571.

Biswal, B., Øren, P.-E., Held, R., Bakke, S., \& Hilfer, R., 2007. Stochastic multiscale model for carbonate rocks, Physical Review E, 75(6), 061303.

Bjørlykke, K., Ramm, M., \& Saigal, G. C., 1989. Sandstone diagenesis and porosity modification during basin evolution, Geologische Rundschau, 78(1), 243-268.

Bloch, S., Lander, R. H., \& Bonnell, L., 2002. Anomalously high porosity and permeability in deeply buried sandstone reservoirs: Origin and predictability, AAPG bulletin, 86(2), 301-328.

Brown, L. T., 2002. Integration of rock physics and reservoir simulation for the interpretation of time-lapse seismic data at Weyburn field, Saskatchewan, Ph.D. thesis, Colorado School of Mines. Arthur Lakes Library. Burnham, K. P. \& Anderson, D. R., 2003. Model selection and multimodel inference: A practical informationtheoretic approach, Springer Science \& Business Media.

Burnham, K. P. \& Anderson, D. R., 2004. Multimodel inference: understanding AIC and BIC in model selection, Sociological methods \& research, 33(2), 261-304.

Cavanaugh, J. E. et al., 1997. Unifying the derivations for the Akaike and corrected Akaike information criteria, Statistics \& Probability Letters, 33(2), 201-208. 
Chapman, M., 2003. Frequency-dependent anisotropy due to meso-scale fractures in the presence of equant porosity, Geophysical prospecting, 51(5), 369-379.

Cilli, P. A. \& Chapman, M., 2020. The power-law relation between inclusion aspect ratio and porosity: Implications for elastic and electrical modeling, Journal of Geophysical Research: Solid Earth, doi:10.1029/2019JB019187.

Cook, R. D., 1977. Detection of influential observation in linear regression, Technometrics, 19(1), 15-18.

Cook, R. D. \& Weisberg, S., 1982. Residuals and influence in regression, New York: Chapman and Hall.

Dvorkin, J. \& Nur, A., 1996. Elasticity of high-porosity sandstones: Theory for two North Sea data sets, Geophysics, 61(5), 1363-1370.

Eberhart-Phillips, D., Han, D.-H., \& Zoback, M. D., 1989. Empirical relationships among seismic velocity, effective pressure, porosity, and clay content in sandstone, Geophysics, 54(1), 82-89.

Eshelby, J. D., 1957. The determination of the elastic field of an ellipsoidal inclusion, and related problems, Proceedings of the Royal Society London A, 241(1226), 376-396.

Fabricius, I. L., 2003. How burial diagenesis of chalk sediments controls sonic velocity and porosity, AAPG bulletin, 87(11), 1755-1778.

Fournier, F., Leonide, P., Biscarrat, K., Gallois, A., Borgomano, J., \& Foubert, A., 2011. Elastic properties of microporous cemented grainstones, Geophysics, 76(6), E211-E226.

Fournier, F., Léonide, P., Kleipool, L., Toullec, R., Reijmer, J. J., Borgomano, J., Klootwijk, T., \& Van Der Molen, J., 2014. Pore space evolution and elastic properties of platform carbonates (Urgonian limestone, Barremian-Aptian, SE France), Sedimentary Geology, 308, 1-17.

Fournier, F., Pellerin, M., Villeneuve, Q., Teillet, T., Hong, F., Poli, E., Borgomano, J., Léonide, P., \& Hairabian, A., 2018. The equivalent pore aspect ratio as a tool for pore type prediction in carbonate reservoirs, AAPG Bulletin, 102(7), 1343.

Gardner, G., Gardner, L., \& Gregory, A., 1974. Formation velocity and density - the diagnostic basics for stratigraphic traps, Geophysics, 39(6), 770-780.

Guilbot, J. \& Smith, B., 2002. 4-D constrained depth conversion for reservoir compaction estimation: Application to Ekofisk field, The Leading Edge, 21(3), 302-308.

Han, D.-h., Nur, A., \& Morgan, D., 1986. Effects of porosity and clay content on wave velocities in sandstones, Geophysics, 51(11), 2093-2107.

Harrigan, T. \& Mann, R., 1984. Characterization of microstructural anisotropy in orthotropic materials using a second rank tensor, Journal of Materials Science, 19(3), 761-767.

Hashin, Z. \& Shtrikman, S., 1963. A variational approach to the theory of the elastic behaviour of multiphase materials, Journal of the Mechanics and Physics of Solids, 11(2), 127-140.

Hill, R., 1952. The elastic behaviour of a crystalline aggregate, Proceedings of the Physical Society. Section A, $\mathbf{6 5}(5), 349$.

Hosa, A. \& Wood, R., 2017. Quantifying the impact of early calcite cementation on the reservoir quality of carbonate rocks: A 3D process-based model, Advances in water resources, 104, 89-104. 
Hurvich, C. M. \& Tsai, C.-L., 1989. Regression and time series model selection in small samples, Biometrika, 76(2), 297-307.

Karol, R. H. \& Berardinelli, C., 2003. Chemical grouting and soil stabilization, CRC Press.

Keehm, Y., 2003. Computational rock physics: Transport properties in porous media and applications, Ph. D. thesis, Stanford University.

Keehm, Y., Mukerji, T., \& Nur, A., 2001. Computational rock physics at the pore scale: Transport properties and diagenesis in realistic pore geometries, The Leading Edge, 20(2), 180-183.

Kuster, G. T. \& Toksöz, M. N., 1974. Velocity and attenuation of seismic waves in two-phase media: Part i. theoretical formulations, Geophysics, 39(5), 587-606.

Latief, F., Biswal, B., Fauzi, U., \& Hilfer, R., 2010. Continuum reconstruction of the pore scale microstructure for Fontainebleau sandstone, Physica A: Statistical Mechanics and its Applications, 389(8), 1607-1618.

Liu, X., Sun, J., \& Wang, H., 2009. Numerical simulation of rock electrical properties based on digital cores, Applied Geophysics, 6(1), 1-7.

Margrave, G. F., 2000. New seismic modelling facilities in MATLAB, Tech. rep., CREWES Research Report 12.

Martys, N. S., Hagedorn, J. G., Goujon, D., \& Devaney, J. E., 1999. Large-scale simulations of single-and multicomponent flow in porous media, in Developments in X-Ray Tomography II, vol. 3772, pp. 205-214, International Society for Optics and Photonics.

Mavko, G., Mukerji, T., \& Dvorkin, J., 2009. The rock physics handbook: Tools for seismic analysis of porous media, Cambridge university press.

McQuarrie, A. D. \& Tsai, C.-L., 1998. Regression and time series model selection, World Scientific.

Moczo, P., Robertsson, J. O., \& Eisner, L., 2007. The finite-difference time-domain method for modeling of seismic wave propagation, Advances in Geophysics, 48, 421-516.

Moore, C. H., 1989. Carbonate diagenesis and porosity, vol. 46, Elsevier.

Mori, T. \& Tanaka, K., 1973. Average stress in matrix and average elastic energy of materials with misfitting inclusions, Acta metallurgica, 21(5), 571-574.

Mousavi, M. A. \& Bryant, S. L., 2012. Connectivity of pore space as a control on two-phase flow properties of tight-gas sandstones, Transport in porous media, 94(2), 537-554.

Mousavi, M. A., Prodanovic, M., \& Jacobi, D., 2012. New classification of carbonate rocks for process-based pore-scale modeling, SPE Journal, 18(02), 243-263.

Nadeau, P. H., 1998. An experimental study of the effects of diagenetic clay minerals on reservoir sands, Clays and Clay Minerals, 46(1), 18-26.

Norris, A. N., 1985. A differential scheme for the effective moduli of composites, Mechanics of materials, 4(1), 1-16.

Pickett, G. R., 1963. Acoustic character logs and their applications in formation evaluation, Journal of Petroleum technology, 15(06), 659-667.

Pyrak-Nolte, L. J., Myer, L. R., \& Cook, N. G., 1990. Transmission of seismic waves across single natural 
fractures, Journal of Geophysical Research: Solid Earth, 95(B6), 8617-8638.

Rahner, M. S., Halisch, M., Fernandes, C. P., Weller, A., \& dos Santos, V. S. S., 2018. Fractal dimensions of pore spaces in unconventional reservoir rocks using x-ray nano-and micro-computed tomography, Journal of Natural Gas Science and Engineering, 55, 298-311.

Reuss, A., 1929. Berechnung der fließgrenze von mischkristallen auf grund der plastizitätsbedingung für einkristalle., ZAMM-Journal of Applied Mathematics and Mechanics/Zeitschrift für Angewandte Mathematik und Mechanik, 9(1), 49-58.

Saenger, E. H., 2008. Numerical methods to determine effective elastic properties, International Journal of Engineering Science, 46(6), 598-605.

Saenger, E. H. \& Bohlen, T., 2004. Finite-difference modeling of viscoelastic and anisotropic wave propagation using the rotated staggered grid, Geophysics, 69(2), 583-591.

Saenger, E. H., Gold, N., \& Shapiro, S. A., 2000. Modeling the propagation of elastic waves using a modified finite-difference grid, Wave motion, 31(1), 77-92.

Sain, R., 2011. Numerical simulation of pore-scale heterogeneity and its effects on elastic, electrical and transport properties, Ph.D. thesis, Stanford University.

Sain, R., Mukerji, T., \& Mavko, G., 2014. How computational rock-physics tools can be used to simulate geologic processes, understand pore-scale heterogeneity, and refine theoretical models, The Leading Edge, 33(3), 324-334.

Saneiyan, S., Ntarlagiannis, D., Werkema Jr, D. D., \& Ustra, A., 2018. Geophysical methods for monitoring soil stabilization processes, Journal of applied geophysics, 148, 234-244.

Singh, J., Curtis, A., Zhao, Y., Cartwright-Taylor, A., \& Main, I., 2019. Coda wave interferometry for accurate simultaneous monitoring of velocity and acoustic source locations in experimental rock physics, Journal of Geophysical Research: Solid Earth, 124(6), 5629-5655.

Stork, A. L., Allmark, C., Curtis, A., Kendall, J.-M., \& White, D. J., 2018. Assessing the potential to use repeated ambient noise seismic tomography to detect $\mathrm{CO} 2$ leaks: Application to the Aquistore storage site, International Journal of Greenhouse Gas Control, 71, 20-35.

Tucker, M. E. \& Wright, V. P., 2009. Carbonate sedimentology, John Wiley \& Sons.

Voigt, W., 1889. Ueber die beziehung zwischen den beiden elasticitätsconstanten isotroper körper, Annalen der physik, 274(12), 573-587.

Wadell, H., 1932. Volume, shape, and roundness of rock particles, The Journal of Geology, 40(5), 443-451.

Walker, D. M., Tordesillas, A., \& Kuhn, M. R., 2016. Spatial connectivity of force chains in a simple shear 3D simulation exhibiting shear bands, Journal of Engineering Mechanics, 143(1), C4016009.

Weil, M. H., DeJong, J. T., Martinez, B. C., \& Mortensen, B. M., 2011. Seismic and resistivity measurements for real-time monitoring of microbially induced calcite precipitation in sand, Geotechnical Testing Journal, 35(2), 330-341.

Wiegmann, A. \& Zemitis, A., 2006. EJ-HEAT: A fast explicit jump harmonic averaging solver for the effective heat conductivity of composite materials. 


\section{J. Singh et al}

Wood, A., 1941. A textbook of sound, Bell, London.

Zhan, X., Schwartz, L. M., Toksöz, M. N., Smith, W. C., \& Morgan, F. D., 2010. Pore-scale modeling of electrical and fluid transport in Berea sandstone, Geophysics, 75(5), F135-F142.

Zimmer, M. A., 2004. Seismic velocities in unconsolidated sands: Measurements of pressure, sorting, and compaction effects, Ph.D. thesis, Stanford University. 